\title{
Synergy Effect of Cooperative Investment
}

\author{
Bogdan Grechuk* Michael Zabarankin ${ }^{\dagger}$
}

\begin{abstract}
Cooperative investment consists of two problems: finding an optimal cooperative investment strategy and fairly dividing investment outcome among participating agents. In general, the two problems cannot be solved separately. It is known that when agents' preferences are represented by mean-deviation functionals, sharing of optimal portfolio creates instruments that, on the one hand, satisfy individual risk preferences but, on the other hand, are not replicable on an incomplete market, so that each agent is strictly better off in participating in cooperative investment than investing alone. This synergy effect is shown to hold when agents' acceptance sets are represented by cash-invariant utility functions in the case of multiperiod investment with an arbitrary feasible investment set. In this case, a set of all Pareto-optimal allocations is characterized, and an equilibrium-based method for selecting a "fair" Pareto-optimal allocation is suggested. It is also shown that if exists, the "fair" allocation belongs to the core of the corresponding cooperative game. The equilibrium-based method is then extended to the case of arbitrary utility functions. The obtained results are demonstrated in a multiperiod cooperative investment problem with investors imposing drawdown constraints on investment strategies.
\end{abstract}

Key Words: cooperative investment, utility function, Pareto-optimal allocation, risk sharing, equilibrium, cooperative game.

\section{Introduction}

Cooperative investment schemes have long been of interest from both theoretical and practical perspectives: in theory, they are often perceived as a branch of the cooperative game theory, whereas in practice, they are commonly implemented and regulated in the form of mutual funds. ${ }^{1}$ Cooperative investment synthesizes three key elements: (i) modeling of agents' risk preferences, (ii) formulating and solving a cooperative investment problem, and (iii) fairly sharing gain/losses of the cooperative investment among the participating agents (investors).

At first glance, cooperative investment seems to offer no advantage over individual investment. Indeed, if agents have different risk preferences, the cooperative portfolio is expected to accommodate all agents' risk constraints and, thus, should be more conservative and should make lesser gains than individual portfolios. So, how can a cooperative portfolio make profit exceeding the sum of the profits from the individual portfolios while conforming to risk preferences of all participating agents? When agents' preferences are represented by mean-deviation functionals, Grechuk et al. [17] showed that the cooperative portfolio does not, in general, accommodate risk preferences of all agents, whereas risk preferences of each agent are satisfied at the stage of fair sharing of the cooperative portfolio's return. However, the exact reason of why cooperative investment has advantage is that agents' shares may not be replicable on an incomplete financial market. In other words, sharing creates instruments that, on one hand, satisfy individual risk preferences but, on the other hand, may not be replicable on the incomplete market, so that each agent is strictly better off in participating in cooperative investment than investing alone. The contribution of this work is showing that this synergy effect also holds when agents' acceptance sets are represented by utility functions and when the portfolio problem is formulated in a multi-period setting with an arbitrary feasible investment set. The following examples demonstrate the synergy effect in two simple cases.

\footnotetext{
* Department of Mathematics, University of Leicester, UK

$\dagger$ Department of Mathematical Sciences, Stevens Institute of Technology, Hoboken, NJ, USA

${ }^{1}$ In [36], a group of agents participating in cooperative investment is called a syndicate.
} 
Example 1 (cash-or-nothing binary options) A cash-or-nothing binary option O pays some fixed amount of cash $\mathrm{C}(\mathrm{O})$ if the option expires in-the-money but nothing otherwise. Suppose a market offers two cash-ornothing binary options $A$ and $B$ for the same price $p$ with $C(A)=2 p$ and $C(B)=3 p$. Then investing a capital $x$ into option $A$ and capital $y$ into option $B$, one earns: $-x-y$ if none of options pays, $x-y$ if only option A pays, $-x+2 y$ if only option B pays, or $x+2 y$ if both options pay. For simplicity, we assume that these four outcomes are equally probable. Thus, investment profits are random variables on a four-element probability space and will be denoted as four-component vectors $X=\left(x_{1}, x_{2}, x_{3}, x_{4}\right)$. Suppose there are two agents with initial capital of $\$ 1$ each. The first one is risk neutral with utility function $U_{1}(X)$ being the expected profit: $U_{1}(X)=\frac{1}{4} \sum_{i=1}^{4} x_{i}$, whereas the second one is risk averse with utility function $U_{2}(X)$ being the second worst outcome of $X$, i.e. if the components of $X$ are ordered as $x_{1} \leqslant x_{2} \leqslant x_{3} \leqslant x_{4}$, then $U_{2}(X)=x_{2}$. Both agents maximize their corresponding utility functions.

Each agent can invest capital $x$ into option $A$ and capital $y=1-x$ into option $B$, which would result in profit $X=(-x-y, x-y,-x+2 y, x+2 y)$. For the first agent, the optimal investment is, obviously, $x=0$ and $y=1$ with the profit $X_{1}^{*}=(-1,-1,2,2)$ for which $U_{1}\left(X_{1}^{*}\right)=E\left[X_{1}^{*}\right]=0.5$. For the second agent, $U_{2}(X)=\min \{x-y,-x+2 y\}$ attains maximum when $x-y=-x+2 y$, so the optimal investment is $x=$ 0.6 and $y=0.4$ with the profit $X_{2}^{*}=(-1,0.2,0.2,1.4)$ for which $U_{1}\left(X_{2}^{*}\right)=0.2$. Now suppose that the agents agree to invest two units of their combined capital into a joint portfolio with $x=0.7$ and $y=2-x=1.3$, and agree to divide the resulting joint profit $X^{*}=(-x-y, x-y,-x+2 y, x+2 y)=(-2,-0.6,1.9,3.3)$ into two shares: $Y_{1}=(-1,-1,1.5,2.9)$ for the first agent and $Y_{2}=X^{*}-Y_{1}=(-1,0.4,0.4,0.4)$ for the second agent. Then $U_{1}\left(Y_{1}\right)=E\left[Y_{1}\right]=0.6>0.5=U_{1}\left(X_{1}^{*}\right)$ and $U_{2}\left(Y_{2}\right)=0.4>0.2=U_{2}\left(X_{2}^{*}\right)$, so each agent has the value of his/her utility function strictly greater than that from optimal individual investment.

Example 2 (single risky instrument) Suppose there is only one risky instrument with a normally disturbed rate of return $r$, having mean $\mu_{r}>0$ and variance $\sigma_{r}^{2}$, available for investment. Also, suppose there are two agents with initial capital of $\$ 1$ each and who would (individually) maximize the expected rate of return of his/her investment into the risky instrument subject to a risk constraint. If agent $i$ invests $x_{i} \in[0,1]$ into the risky instrument and keeps $1-x_{i}$ in cash, then his/her portfolio rate of return is determined by $x_{i} r$, where $i=1$, 2. Then agent 1 measures risk by standard deviation and imposes a constraint $\sigma\left(x_{1} r\right) \leqslant \sigma_{0}$ (with $\left.\sigma_{0}<\sigma_{r}\right)$, whereas agent 2 requires that the probability of $x_{2} r$ to drop below $E\left[x_{2} r\right]-\Delta$ should not exceed $\alpha$ for specified $\Delta \in\left(0, \mu_{r}-q_{\alpha}(r)\right]$ and $\alpha<1 / 2$, where $q_{\alpha}(X)$ is the $\alpha$-quantile of a random variable $X$. Specifically, agents 1 and 2 would solve the following individual investment problems

$$
\max _{x_{1} \in[0,1]} E\left[x_{1} r\right] \quad \text { subject to } \sigma\left(x_{1} r\right) \leqslant \sigma_{0}
$$

and

$$
\max _{x_{2} \in[0,1]} E\left[x_{2} r\right] \quad \text { subject to } \quad \mathbb{P}\left[x_{2} r<E\left[x_{2} r\right]-\Delta\right] \leqslant \alpha,
$$

respectively. Since each problem has only one variable and at optimality, the constraints on portfolio risks hold as equalities, optimal $x_{1}$ and $x_{2}$ are determined by $x_{1}^{*}=\sigma_{0} / \sigma_{r}$ and $x_{2}^{*}=\Delta /\left(\mu_{r}-q_{\alpha}(r)\right)$, respectively. Thus, agents 1 and 2 would obtain expected rates of return of $\mu_{r} \sigma_{0} / \sigma_{r}$ and $\mu_{r} \Delta /\left(\mu_{r}-q_{\alpha}(r)\right)$, respectively.

Now suppose the two agents agree to invest joint capital of $x \in[0,2]$ into the risky instrument and then to divide the random rate of return $Y=x r$ into shares $Y_{1}$ and $Y_{2}$ such that $Y_{1}+Y_{2} \equiv Y$ and

$$
Y_{1}=\phi_{\alpha}(Y)-C, \quad Y_{2}=Y-\left(\phi_{\alpha}(Y)-C\right),
$$

where $\alpha<1 / 2, C$ is a constant to be determined, and $\phi_{\alpha}(Y)$ is a random variable defined by

$$
\phi_{\alpha}(Y)=\left\{\begin{array}{cl}
Y & \text { if } q_{\alpha}(Y) \leqslant Y \leqslant q_{1-\alpha}(Y) \\
E[Y], & \text { otherwise. }
\end{array}\right.
$$


The shares satisfy corresponding risk constraints: $\sigma\left(Y_{1}\right) \leqslant \sigma_{0}$ and $\mathbb{P}\left[Y_{2}<E\left[Y_{2}\right]-\Delta\right] \leqslant \alpha$, provided that $x \leqslant \sigma_{0} /\left(\xi_{\alpha} \sigma_{r}\right)$, where $\xi_{\alpha}=\sigma\left(\phi_{\alpha}(Z)\right)$ with $Z$ being the standard normal variable. Thus, the maximum expected rate of return of the cooperative investment is $\mu_{r} \sigma_{0} /\left(\xi_{\alpha} \sigma_{r}\right)$ with optimal $x$ to be $x^{*}=\sigma_{0} /\left(\xi_{\alpha} \sigma_{r}\right)$. If $\Delta<\sigma_{0}\left(1-\xi_{\alpha}\right) /\left(\xi_{\alpha} \Phi^{-1}(1-\alpha)\right)$, where $\Phi^{-1}(\cdot)$ is the inverse of the CDF of $Z$, then $E\left[x^{*} r\right]>E\left[x_{1}^{*} r\right]+$ $E\left[x_{2}^{*} r\right]$, and the constant $C$ can be chosen such that $E\left[Y_{i}\right]>E\left[x_{i}^{*} r\right], i=1,2$, so that each agent is strictly better off in cooperative investment.

Both examples show that agents' shares $Y_{1}$ and $Y_{2}$ of the optimal cooperative investment neither exist on the market nor can be replicated with the available market instruments. This is exactly where cooperative investment gains synergy on an incomplete market. In fact, the set of contingent claims accessible by agents through cooperative investment is much larger than the sets that they can reach in their own. As a result, cooperative investment would almost always improve agents' individually optimal investments.

The examples also reveal that forming a cooperative portfolio and fair sharing are not independent problemsthe agents agree to participate in cooperative investment only if they are guaranteed to have shares that outperform their individual investments.

A cooperative investment problem is formulated as follows. Suppose there are $m$ agents, whose risk/reward preferences are described by utility functions $U_{i},{ }^{2} i=1, \ldots, m$, and let $\mathcal{F}$ be a feasible set of rates of return from available instruments, including their combinations and possibly their derivatives. If $Y_{i}$ is a share of agent $i$ of the rate of return of a cooperative portfolio, then cooperative investment is an $m$-criteria optimization problem

$$
\max _{\sum_{i=1}^{m} Y_{i} \in \mathcal{F}}\left\{U_{1}\left(Y_{1}\right), \ldots, U_{m}\left(Y_{m}\right)\right\}
$$

whose solution is a feasible Pareto-optimal vector $\left(Y_{1}, \ldots, Y_{m}\right)$, i.e. $\sum_{i=1}^{m} Y_{i} \in \mathcal{F}$ and there is no $\left(Y_{1}^{\prime}, \ldots, Y_{m}^{\prime}\right)$ such that $\sum_{i=1}^{m} Y_{i}^{\prime} \in \mathcal{F}$ and $U_{i}\left(Y_{i}^{\prime}\right) \geqslant U_{i}\left(Y_{i}\right), i=1, \ldots, m$, with at least one inequality being strict. For the extension of notion of Pareto-optimality for agents with different beliefs, see [15].

Typically, an investor describes his/her risk-reward preferences to a fund manager in the form of rules and restrictions rather than in terms of a specific utility function. This implies that the investor has an acceptance set $\mathcal{A}$ such that a random outcome $X$ is desired if $X \in \mathcal{A}$. In fact, a utility function corresponding to such an acceptance set can be readily determined by $U(X)=\sup \{C \mid X-C \cdot e \in \mathcal{A}\}$, where $e$ is a numeraire with $\mathbb{P}[e=1]=1$. In other words, $U(X)$ is the amount of "cash" in units of $e$ that should be added to a random outcome $X$ in order to be accepted. It is straightforward to verify that $U$ is cash invariant: $U(X+C)=$ $U(X)+C$ for any constant $C$. This practical aspect is one of the key reasons for distinguishing the class of cashinvariant utility functions: if investor's rules and restrictions can be formalized in the form of mathematically tractable and feasible constraints, they always have a numerical representation in the form of a cash-invariant utility function. In other words, the set of investor's rules/restrictions may or may not agree with rational axioms of a preference relation, e.g. transitivity, monotonicity, continuity, etc., and the investor's cash-invariant utility is merely a convenient numerical representation of the investor's acceptance set.

Of course, the structure of problem (1) is well known. What makes difference, though, is the assumptions on $\mathcal{F}$ and $U_{i}$ under which (1) has been studied. For example, if $\mathcal{F}=\{X\}$ is a singleton, and also if $X=\sum_{i=1}^{m} Q_{i}$, where $Q_{i}$ is an initial endowment of agent $i$, problem (1) reduces to "traditional" optimal risk sharing, which is a well-studied problem. In particular, it was studied with

- Expected utilities $U_{i}$ (see $\left.[6,14,1]\right)$,

- Convex risk measures ${ }^{3}$ in place of $U_{i}$ (see $[11,13,23,4,24]$ ), and

- Mean-deviation functionals ${ }^{4} U_{i}$ (see [21]).

\footnotetext{
${ }^{2}$ Given two instruments with random payoffs $X_{1}$ and $X_{2}$, agent $i$ prefers $X_{1}$ if and only if $U_{i}\left(X_{1}\right)>U_{i}\left(X_{2}\right)$.

${ }^{3}$ For extension to the non-monotone case, see [2].

${ }^{4}$ Mean-deviation functionals combine two quantities: expected value and general deviation measure of a random variable.
} 
For all classes of utility functions listed above, a characterization of Pareto-optimal solutions to risk sharing version of (1) has been obtained, and tractable sufficient conditions for solution existence have been developed, see [1] for a detailed survey.

However, much less is known in the case when $\mathcal{F}$ in (1) is not a singleton. For the expected utility case, this problem was studied in [36,37]. Recently, Grechuk et al. [17] solved problem (1) for $U_{i}$ given by meandeviation functionals and for $\mathcal{F}$ being a set of rates of returns of all possible linear combinations of a finite number of instruments available on the market.

This work extends $[37,17]$ as well as $[1,23,21]$ in two directions:

(i) The "investment set" $\mathcal{F}$ is not confined to consist of random variables, representing the rates of return of linear combinations of predetermined instruments-it may consist, for example, of stochastic processes, representing the whole price evolution resulting from dynamic trading strategies;

(ii) $U_{i}$ are arbitrary utility functions (not necessarily convex), though, special consideration is given to cashinvariant utilities.

If all $U_{i}$ are cash-invariant, then there exists a cooperative utility function $U^{*}$ that represents "integral" risk/reward preferences of all agents participating in cooperative investment, and problem (1) reduces to two independent problems:

(i) Finding optimal cooperative portfolio from $\max _{X \in \mathcal{F}} U^{*}(X)$, and

(ii) Fairly sharing $X^{*}=\operatorname{argmax}_{X \in \mathcal{F}} U^{*}(X)$ among the participating agents.

This case yields three closely related results: complete characterization of the set of all Pareto-optimal solutions to (1); an equilibrium-based method for selecting a "fair" solution from the Pareto-optimal set; and showing that the fair solution belongs to the core of the corresponding cooperative game with transferable payoffs.

If $U_{i}$ are not cash-invariant, finding an optimal cooperative investment and selecting a "fair" Pareto-optimal allocation may not be separable. This problem is resolved by introducing an equilibrium pricing functional $P$, consistent with "external" market prices. Showing existence of such $P$ reduces to the well-studied question of equilibria existence in classical risk sharing. In addition, if $P$ exists and unique, it can be used for assigning a unique "fair" price to every contingent claim in an incomplete market. As an illustration, the obtained results are applied to a cooperative investment problem with agents who impose constraints on investment drawdowns in a multiperiod model.

The paper is organized into six sections. Section 2 formulates a general cooperative investment problem and discusses several motivating examples. Section 3 solves the problem with cash-invariant utility functions, whereas Section 4 addresses the case with arbitrary utility functions. Section 5 demonstrates cooperative investment for investors using drawdown risk measure. Section 6 concludes the work.

\section{Problem Formulation}

In an abstract setting, an optimal investment problem can be formulated by

$$
\sup _{X \in \mathcal{F}} U(X)
$$

where $\mathcal{F}$ is a non-empty subset of a locally convex topological vector space $\mathcal{X}$, and $U: \mathcal{X} \rightarrow[-\infty, \infty)$. Elements $X \in \mathcal{X}$ will be associated with investments, $\mathcal{F}$ is a set of all feasible $X$, and $\mathcal{U}$ is a utility function. By "typical setting" will be meant the case $\mathcal{X}=\mathcal{L}^{p}(\Omega)=\mathcal{L}^{p}(\Omega, \mathcal{M}, \mathbb{P}), p \in[1, \infty]$, where $(\Omega, \mathcal{M}, \mathbb{P})$ is a probability space, with $\Omega$ denoting the designated space of future states $\omega, \mathcal{M}$ is a field of sets in $\Omega$, and $\mathbb{P}$ is a probability measure on $(\Omega, \mathcal{M})$ such that all investors agree on it. ${ }^{5}$ In this case, elements $X \in \mathcal{X}$ are random

\footnotetext{
${ }^{5}$ This is the so-called "common belief" assumption.
} 
variables (r.v.'s), which will be interpreted as discounted gains from investments, and $\mathcal{F} \subset \mathcal{X}$ are discounted gains of feasible investments.

Let $I=\{1,2, \ldots, m\}$ be the set of agents, who can either invest in the market individually or can form a cooperative portfolio $X \in \mathcal{F}$ and divide it into shares $Y_{i}, i \in I$, so that $X=\sum_{i=1}^{m} Y_{i}$. Let $U_{i}: \mathcal{X} \rightarrow[-\infty, \infty)$ be utility function of agent $i \in I$. If $\mathcal{F}$ is a subspace of $\mathcal{X}$, a trivial strategy is to form portfolio $X^{*}=\sum_{i=1}^{m} Y_{i}^{*}$, where

$$
Y_{i}^{*}=\underset{X \in \mathcal{F}}{\operatorname{argmax}} U_{i}(X), \quad i \in I,
$$

and to divide $X^{*}$ by assigning $Y_{i}^{*}$ to agent $i$. We will show that, in general, this strategy may not be optimal.

By cooperative investment will be meant triplet $\mathcal{P}=(\mathcal{X}, \mathcal{F}, \mathbf{U})$ with utility functions $\mathbf{U}=\left(U_{1}, \ldots, U_{m}\right)$. Any vector $\mathbf{Y}=\left(Y_{1}, \ldots, Y_{m}\right)$ with $Y_{i} \in \mathcal{X}$ for all $i \in I$ will be called allocation. An allocation $\mathbf{Y}$ is $\mathcal{F}$-feasible if $\sum_{i=1}^{m} Y_{i} \in \mathcal{F}$. $\mathcal{F}$-feasible allocations $\mathbf{Y}=\left(Y_{1}, \ldots, Y_{m}\right)$ and $\mathbf{Z}=\left(Z_{1}, \ldots, Z_{m}\right)$ are equivalent if $U_{i}\left(Y_{i}\right)=$ $U_{i}\left(Z_{i}\right), i \in I$ (equivalent allocations will not be distinguished). $\mathbf{Z}$ dominates $\mathbf{Y}$ if $U_{i}\left(Z_{i}\right) \geqslant U_{i}\left(Y_{i}\right), i \in I$, with at least one inequality being strict. An $\mathcal{F}$-feasible allocation $\mathbf{Y}$ is $\mathcal{F}$-Pareto optimal if no $\mathcal{F}$-feasible allocation dominates $\mathbf{Y}$.

The following example shows that individual investments may not form an $\mathcal{F}$-Pareto optimal allocation.

Example 3 (typical setting) In the "typical setting," assume that

(a) All agents are strictly risk averse. That is, if $E[u(X)]>E[u(Y)]$ for all strictly concave $u: \mathbb{R} \rightarrow \mathbb{R}$, then $U_{i}(X)>U_{i}(Y), i \in I$; and

(b) Not all $Y_{i}^{*}$ are pairwise comonotone. ${ }^{6}$

Then vector $\mathbf{Y}=\left(Y_{1}^{*}, \ldots, Y_{m}^{*}\right)$ is not $\mathcal{F}$-Pareto optimal.

Detail. An r.v. $X$ is said to dominate an r.v. $Y$ by convex order, if $E[u(X)] \geqslant E[u(Y)]$ for all concave $u: \mathbb{R} \rightarrow \mathbb{R}$. By [28], there exist pairwise comonotone r.v.'s $Z_{1}, \ldots, Z_{m}$ such that $\sum_{i=1}^{m} Z_{i}=\sum_{i=1}^{m} Y_{i}^{*}$, and $Z_{i}$ dominates $Y_{i}^{*}$ by convex order, $i=1, \ldots, m$. From (b) and the proof in [28], it also follows that, for some $i$, the distributions of $Z_{i}$ and $Y_{i}^{*}$ are different, which implies that $E\left[u\left(Z_{i}\right)\right]>E\left[u\left(Y_{i}^{*}\right)\right]$ for all strictly concave $u$, hence $U_{i}\left(Z_{i}\right)>U_{i}\left(Y_{i}^{*}\right)$ by (a). For other indices $i$, we either have $U_{i}\left(Z_{i}\right)>U_{i}\left(Y_{i}^{*}\right)$ by a similar argument, or the distributions of $Z_{i}$ and $Y_{i}^{*}$ are the same, and then $U_{i}\left(Z_{i}\right)=U_{i}\left(Y_{i}\right)$.

Because agents are assumed to be risk averse in most applications, and because there is no reason to expect that the rates of return $Y_{i}^{*}$ of optimal portfolios of agents $i=1, \ldots, m$ happen to be all pairwise comonotone, Example 3 demonstrates that "typically" individual investment is not a Pareto optimal strategy, that is, cooperative investment is strictly profitable for at least one investor. The following proposition shows that, in such cases, cooperative investment may be strictly profitable for all investors.

Proposition 1 Suppose all $\mathcal{U}_{i}, i \in I$, are continuous and have no local maxima, and some $\mathcal{F}$-feasible allocation $\mathbf{Y}$ is not $\mathcal{F}$-Pareto optimal. Then there exists an $\mathcal{F}$-feasible allocation $\mathbf{Z}$ such that $U_{i}\left(Z_{i}\right)>U_{i}\left(Y_{i}\right), i \in I$.

Proof Because $\mathbf{Y}$ is not $\mathcal{F}$-Pareto optimal, there exists an $\mathcal{F}$-feasible allocation $\mathbf{Z}^{\prime}$ such that $U_{i}\left(Z_{i}^{\prime}\right) \geqslant U_{i}\left(Y_{i}\right), i \in$ $I$, with at least one inequality being strict. We may assume that $U_{1}\left(Z_{1}^{\prime}\right)>U_{1}\left(Y_{1}\right)$. Because $U_{i}, i \in I$, have no local maxima, there exist sequences $Z_{i}^{(n)}$ such that $\lim _{n \rightarrow \infty} Z_{i}^{(n)}=Z_{i}^{\prime}, i \in I$, and $U_{i}\left(Z_{i}^{(n)}\right)>U_{i}\left(Z_{i}^{\prime}\right), i \in I$, $n \in \mathbb{N}$. Then $\lim _{n \rightarrow \infty}\left(Z_{1}^{\prime}+\sum_{i=2}^{m}\left(Z_{i}^{\prime}-Z_{i}^{(n)}\right)\right)=Z_{1}^{\prime}$, hence, by continuity of $U_{1}, U_{1}\left(Z_{1}^{\prime}+\sum_{i=2}^{m}\left(Z_{i}^{\prime}-Z_{i}^{(n)}\right)\right)>$ $U_{1}\left(Y_{1}\right)$ for some $n$. Now, the statement of proposition holds with $Z_{1}=Z_{1}^{\prime}+\sum_{i=2}^{m}\left(Z_{i}^{\prime}-Z_{i}^{(n)}\right), Z_{i}=Z_{i}^{(n)}$, $i=2, \ldots, m$.

This work addresses the following questions.

${ }^{6}$ Two r.v.'s $X: \Omega \rightarrow \mathbb{R}$ and $Y: \Omega \rightarrow \mathbb{R}$ are said to be comonotone, if there exists a set $A \subset \Omega$ such that $P[A]=1$ and $\left(X\left(\omega_{1}\right)-X\left(\omega_{2}\right)\right)\left(Y\left(\omega_{1}\right)-Y\left(\omega_{2}\right)\right) \geqslant 0$ for all $\omega_{1}, \omega_{2} \in A$. 
(Q1) How to determine optimal cooperative investment $X \in \mathcal{F}$ ?

(Q2) How to find an $\mathcal{F}$-Pareto optimal allocation of $X$ ?

(Q3) If $\mathcal{F}$-Pareto optimal allocation is not unique, what is a "fair" one?

The "typical setting" $\mathcal{X}=\mathcal{L}^{p}(\Omega)$ includes the following examples.

Example 4 (one period model) Suppose there are $n+1$ assets with the rates of return $r_{0}, r_{1}, \ldots, r_{n}$, where asset 0 is assumed to be riskless. An agent forms a portfolio $\mathbf{x}=\left(x_{0}, x_{1}, \ldots, x_{n}\right)$, where $x_{j}$ is the amount of money invested into asset $j, j=0,1, \ldots, n$, so that $C=\sum_{j=0}^{n} x_{j}$ is the initial capital. The portfolio gain $X(\mathbf{x})$ is then defined as $X(\mathbf{x})=\frac{1}{1+r_{0}} \sum_{j=0}^{n}\left(1+r_{j}\right) x_{j}-C=\frac{1}{1+r_{0}} \sum_{j=1}^{n}\left(r_{j}-r_{0}\right) x_{j}$. If borrowing and shorting are allowed, the budget constraint can be eliminated by expressing $x_{0}=C-\sum_{j=1}^{n} x_{j}$. The set of discounted gains of feasible portfolios is then defined as $\mathcal{F}=\left\{X \mid X=\frac{1}{1+r_{0}} \sum_{j=1}^{n}\left(r_{j}-r_{0}\right) x_{j}, \quad\left(x_{1}, \ldots, x_{n}\right) \in \mathbb{R}^{n}\right\}$.

Example 5 (multiperiod model) Suppose $n+1$ assets are traded at time moments $t=0,1, \ldots, T$. A price vector $\mathbf{S}_{t}=\left(S_{t}^{0}, S_{t}^{1}, \ldots, S_{t}^{n}\right)$, where $S_{t}^{i} \in \mathcal{L}^{p}(\Omega)$ is the price of asset $i$ at time $t$, is measurable with respect to $\sigma$-algebra $\mathcal{M}_{t} \subset \mathcal{M}$, where $\mathcal{M}_{0} \subset \mathcal{M}_{1} \subset \cdots \subset \mathcal{M}_{T}$. A trading strategy is $\mathbb{R}^{n+1}$-valued process $\mathbf{x}_{t}=\left(x_{t}^{0}, x_{t}^{1}, \ldots, x_{t}^{n}\right), t=1, \ldots, T$, such that $x_{t}^{i}$ is $\mathcal{M}_{t-1}$ measurable. A trading strategy $\mathbf{x}_{t}$ is called selffinancing if $\mathbf{x}_{t} \cdot \mathbf{S}_{t}=\mathbf{x}_{t+1} \cdot \mathbf{S}_{t}$ for $t=1, \ldots, T-1$. The discounted portfolio value at time $t$ in terms of numeraire 0 is defined as $X_{t}=\mathbf{x}_{t} \cdot \mathbf{S}_{t} / S_{t}^{0}$. The set of discounted gains of feasible portfolios is then $\mathcal{F}=$ $\left\{X_{T}(\mathbf{x})-X_{0}(\mathbf{x}) \mid \mathbf{x}_{t}\right.$ is self-financing $\}$.

Example 6 (complete market) Assume that every contingent claim $Y \in \mathcal{L}^{p}(\Omega)$ is attainable, that is, available on the market for some price $P(Y)=E_{Q}[Y]$, where $Q$ is a probability measure equivalent to $\mathbb{P}$. If the number of assets is finite, and the probability space $(\Omega, \mathcal{M}, \mathbb{P})$ is atomless ${ }^{7}$, this is possible only in continuous-time market models. The set $\mathcal{F}$ of portfolio gains is then $\mathcal{F}=\left\{Y-E_{Q}[Y]\right\}=\left\{X \mid E_{Q}[X]=0\right\}$.

In all the examples above, $\mathcal{F}$ is a subspace of $\mathcal{X}=\mathcal{L}^{p}(\Omega)$. This is not the case, if, for example, shorting is not allowed. More generally, the "typical setting" may not be sufficient for market models with additional assumptions.

Example 7 (transaction costs) In models with transaction costs, a portfolio does not have exact equivalent in terms of a single numeraire, therefore the portfolio value (or gain) is often expressed as an n-component vector, where $n$ is the number of assets, see [31]. In particular, a natural outcome of any trading strategy in a dollar-euro currency exchange market is a 2-dimensional random vector $(X, Y)$, where $X$ and $Y$ denotes the gain in dollars and euros, correspondingly.

Example 8 (path-dependent utility) In multi-period or continuous time market models, an agent's utility function may depend on the whole price evolution rather than on a final payoff. In this case, the elements of $\mathcal{X}$ are stochastic processes.

All these five examples are special cases of problem (2). Also, a portfolio problem

$$
\sup _{X \in \mathcal{F}} U(Q+X),
$$

with an initial endowment $Q \in \mathcal{X}$ can be reduced to (2) by introducing $\widetilde{U}(X)=U(Q+X)$, so we can assume that there is no initial endowment.

Often, agent's preferences are described by a consumption set $\mathcal{X}^{\prime} \subset \mathcal{X}$ with a utility function $U^{\prime}: \mathcal{X}^{\prime} \rightarrow$ $\mathbb{R}$. This is equivalent to the set $\mathcal{X}^{\prime}=\{X \mid U(X)>-\infty\}$. In the "typical setting," examples of frequently used utility functions include

\footnotetext{
${ }^{7} \mathrm{~A}$ probability space is atomless, if there exists an r.v. with continuous cumulative distribution function.
} 
(i) von Neumann-Morgenstern expected utility functions. Let $U(X)=E[u(X)]$, where $u: \mathbb{R} \rightarrow \mathbb{R}$ is a strictly increasing and strictly concave function, such that the expectation is well-defined. A popular example is exponential utility function given by $u(x)=1-e^{-\alpha x}, \alpha>0$. If, for some r.v.'s $X, Y \in$ $\mathcal{L}^{p}(\Omega), E[u(X)] \geqslant E[u(Y)]$ for every increasing and concave $u$, we say that $X$ dominates $Y$ by second order stochastic dominance (SSD), and write $X \succeq_{2} Y$. In general, any utility function $U: \mathcal{L}^{p}(\Omega) \rightarrow \mathbb{R}$ is called consistent with $S S D$ if $U(X) \geqslant U(Y)$ whenever $X \succeq_{2} Y$.

(ii) Expected utility functions with constraints. Let $\mathcal{X}^{\prime}=\{X \mid P(X>-C)=1\}$ for some constant $C$, and let $U^{\prime}(X)=E\left[u^{\prime}(X)\right]$, where $u^{\prime}:[-C, \infty) \rightarrow \mathbb{R}$ is a strictly increasing and strictly concave function. A popular example is an HARA utility function defined by $u(x)=\frac{1}{\alpha}(x+C)^{\alpha}$ for $\alpha \in(0,1)$ and by $u(x)=\ln (x+C)$ for $\alpha=0$.

(iii) Monetary utility functions. A utility function $U: \mathcal{L}^{p}(\Omega) \rightarrow[-\infty, \infty)$ is called

(U1) cash invariant, if $u(X+C)=u(X)+C, X \in \mathcal{L}^{p}(\Omega), C \in \mathbb{R}$;

(U2) monotone, if $U(X) \geqslant U(Y)$ whenever $P(X \geqslant Y)=1$;

(U3) concave, if $U(\lambda X+(1-\lambda) Y) \geqslant \lambda U(X)+(1-\lambda) U(Y)$ for $0 \leqslant \lambda \leqslant 1$;

(U4) upper semicontinuous, if set $\left\{X \in \mathcal{L}^{p}(\Omega) \mid U(X) \geqslant C\right\}$ is closed for all $C>-\infty$;

(M) monetary utility function if U1-U4 holds.

A utility function $U$ satisfies $U 1$ if and only if it can be represented by $U(X)=\sup \{C \mid X-C \in \mathcal{A}\}$, where $\mathcal{A}=\{X \mid U(X) \geqslant 0\}$ is called acceptance set. In this case, $U$ is interpreted as the maximal amount of cash $C$ one can consume such that the remaining payoff $X-C$ is still in the acceptance set. U2 is an assumption that "more is better," and U3 manifests the diversification principle: a mixture $\lambda X+(1-\lambda) Y$ cannot be worse than each of $X$ and $Y$, or that

(U3') $U(\lambda X+(1-\lambda) Y) \geqslant \min \{U(X), U(Y)\}$ for $0 \leqslant \lambda \leqslant 1$.

U3' is equivalent to U3 provided U1 [5, Proposition 2.1]. A utility function $U: \mathcal{L}^{p}(\Omega) \rightarrow \mathbb{R}$ is called law-invariant if $U(X)=U(Y)$ whenever $X$ and $Y$ have the same distribution. Under a minor technical assumption, ${ }^{8}$ every law-invariant monetary utility function is consistent with SSD [14]. If $U$ is a monetary utility function, $\mathcal{R}(X)=-U(X)$ is a convex risk measure, see [14]. In fact, investor's risk measure can be determined as a solution of the inverse portfolio optimization problem provided that the rate of return of investor's either optimal or preferable portfolio is given (known), see [19].

(iv) Mean-variance analysis. Markowitz-type portfolio selection problems [30]

$$
\inf _{X \in \mathcal{F}, E[X] \geqslant \pi} \sigma(X) \text { and } \sup _{X \in \mathcal{F}, \sigma(X) \leqslant \sigma_{0}} E[X]
$$

where $\sigma(X)=\|X-E[X]\|_{2}$, are special cases of (2) with a utility function $U$ defined in the second case by $U(X)=E[X]$ for $\sigma(X) \leqslant \sigma_{0}$ and by $U(X)=-\infty$ for $\sigma(X)>\sigma_{0}$.

(v) Mean-deviation analysis. Rockafellar et al. [33] replaced standard deviation $\sigma(X)$ in (5) by a general deviation measure, which is defined as a functional $\mathcal{D}: \mathcal{L}^{p}(\Omega) \rightarrow[0 ; \infty]$ satisfying axioms

(D1) $\mathcal{D}(X)=0$ for constant $X$, but $\mathcal{D}(X)>0$ otherwise (nonnegativity),

(D2) $\mathcal{D}(\lambda X)=\lambda \mathcal{D}(X)$ for all $X$ and all $\lambda>0$ (positive homogeneity),

(D3) $\mathcal{D}(X+Y) \leqslant \mathcal{D}(X)+\mathcal{D}(Y)$ for all $X$ and $Y$ (subadditivity),

\footnotetext{
${ }^{8}$ Namely, this holds if $\Omega$ is atomless and $\mathcal{L}^{2}(\Omega)$ is separable.
} 
(D4) set $\left\{X \in \mathcal{L}^{2}(\Omega) \mid \mathcal{D}(X) \leqslant C\right\}$ is closed for all $C<\infty$ (lower semicontinuity).

Examples of deviation measures include standard lower semideviation $\sigma_{-}(X)=\left\|[X-E[X]]_{-}\right\|_{2}$, where $[X]_{-}=\max \{0,-X\}$, mean absolute deviation $\operatorname{MAD}(X)=\|X-E[X]\|_{1}$, etc. see [33]. A corresponding utility function in (2) can be determined by

$$
U(X)=\left\{\begin{array}{lll}
E[X] & \text { when } & \mathcal{D}(X) \leqslant d, \\
-\infty & \text { when } & \mathcal{D}(X)>d,
\end{array}\right.
$$

for some $d>0$. As with coherent risk measures, investor's deviation measure can be recovered from the rate of return of investor's optimal (preferable) portfolio, see [20].

(vi) Value-at-risk(VaR). For an r.v. $X$, the three functions $F_{X}(x)=\mathbb{P}[X \leqslant x], q_{\alpha}(X)=\inf \left\{x \mid F_{X}(x)>\alpha\right\}$, and $\operatorname{VaR}_{\alpha}(X)=-q_{\alpha}(X)$ are cumulative distribution function (CDF), quantile function, and value at risk, respectively. Despite lacking convexity, VaR remains arguably the most popular risk measure in banking industry. The related utility functions are defined by

(a) $U(X)=-\operatorname{VaR}_{\alpha}(X)=q_{\alpha}(X)$ for $\alpha \in(0,1)$,

(b) $U(X)=1-\mathbb{P}[X<C]=\mathbb{P}[X \geqslant C], C \in \mathbb{R}$;

(c) Chance constraint: an agent maximizes $E[X]$ subject to the constraint $\mathbb{P}[E[X]-X>\Delta] \leqslant \alpha$ for $\alpha \in(0,1)$ and $\Delta>0$ (the probability that the loss $E[X]-X$ exceeds the threshold $\Delta$ is bounded by $\alpha$ ) or, equivalently, $\operatorname{VaR}_{\alpha}(X-E[X]) \leqslant \Delta$. A corresponding utility function is given by

$$
U(X)=\left\{\begin{array}{lll}
E[X] & \text { when } & \operatorname{VaR}_{\alpha}(X-E[X]) \leqslant \Delta, \\
-\infty & \text { when } \operatorname{VaR}_{\alpha}(X-E[X])>\Delta .
\end{array}\right.
$$

The next and last example is beyond the "typical setting."

(vii) Central to active portfolio management is the notion of drawdown: investors close their accounts if a hedge fund is either in a single large drawdown (typically 20\%) or in a small but prolonged drawdown (over a year) $[8,9,39]$. As a result, fund drawdowns are closely monitored and imposed various constraints. However, sequences of fund drawdowns can not be easily compared even for the same time periods. They are often converted into a single number called drawdown measure, which, for example, can be either the maximum or average drawdown over a specified period. In fact, a popular drawdown measure is conditional drawdown-at-risk $(C D a R)$ with confidence level $\alpha \in[0,1]$, which is defined as the average of $(1-\alpha) 100 \%$ largest drawdowns over a specified time period and includes the maximum and average drawdowns as special cases corresponding to $\alpha=1$ and $\alpha=0$, respectively. A typical investment problem is maximizing the portfolio terminal value subject to a constraint on the portfolio $\mathrm{CDaR}$ over the whole investment horizon; see [8, 9, 39]. Formulation of this multiperiod investment problem as utility maximization is as follows.

Let $\mathcal{X}$ be a space of stochastic processes $X=X_{t}, t \in \mathcal{T}$, where $\mathcal{T}$ is either $\left\{t_{1}, t_{2}, \ldots, t_{k}=T\right\}$ (multi-period model) or [0,T] (continuous-time model). For a fixed $\omega \in \Omega$ and $t \in \mathcal{T}$, let $d_{\omega, t}(X)=$ $\sup _{\tau \leqslant t} X_{\tau}(\omega)-X_{t}(\omega)$ be drawdown of $X$; see [22, 10, 8, 9, 39]. If $t \in \mathcal{T}$ is chosen uniformly at random, $d(X)=d_{\omega, t}(X)$ can be interpreted as a random variable on $\Omega \times \mathcal{T}$ with quantile function ${ }^{9}$ $q_{s}(d(X))$. CDaR with confidence level $\alpha \in[0,1]$ is defined by $[8,9,39]$

$$
\mathrm{CDaR}_{\alpha}(X)=\frac{1}{1-\alpha} \int_{\alpha}^{1} q_{s}(d(X)) d s, \quad \alpha \in(0,1),
$$

\footnotetext{
${ }^{9}$ Formally, we first introduce a probability measure $\mathbb{P}_{\mathcal{T}}$ on $\mathcal{T}$ such that $\mathbb{P}_{\mathcal{T}}\left(t_{i}\right)=1 / k, i=1, \ldots, k$, if $\mathcal{T}=\left\{t_{1}, t_{2}, \ldots, t_{k}=T\right\}$, and $\mathbb{P}_{\mathcal{T}}(S)=l(S) / T$ if $\mathcal{T}=[0, T]$, where $S$ is any measurable subset of $[0, T]$ with Lebesgue measure $l(S)$, and then introduce a probability measure on $\Omega \times \mathcal{T}$ as a produce measure of $\mathbb{P}$ and $\mathbb{P}_{\mathcal{T}}$.
} 
where the limiting cases $\operatorname{MaxDD}(X) \equiv \lim _{\alpha \rightarrow 1} \mathrm{CDaR}_{\alpha}(X)=\sup d(X)$ and $\operatorname{AvgDD}(X) \equiv \mathrm{CDaR}_{0}(X)$ are the maximum drawdown and the average drawdown, respectively. The problem of maximizing the terminal expected value $E\left[X_{T}\right]$ subject to a constraint $\mathrm{CDaR}_{\alpha}(X) \leqslant C$ for some $C>0$ (see $[8,9,39]$ ) can be represented in the form of (2) with the utility function

$$
U(X)=\left\{\begin{array}{lll}
E\left[X_{T}\right] & \text { when } & \operatorname{CDaR}_{\alpha}(X) \leqslant C \\
-\infty & \text { when } & \operatorname{CDaR}_{\alpha}(X)>C
\end{array}\right.
$$

Questions Q1-Q3 were answered in [17] for problem (1) with $U_{i}$ given by mean-deviation functionals and with $\mathcal{F}$ defined as in Example 4. Next section studies these questions in the more general settings.

\section{Cooperative Investment}

We will focus of studying problem (1) with cash invariant utility functions. The case of arbitrary utility functions is discussed in Section 4. A utility function $U_{i}: \mathcal{X} \rightarrow[-\infty, \infty)$ is cash invariant, if there exists an $e \in \mathcal{X}$ such that

$$
U_{i}(X+C \cdot e)=U_{i}(X)+C, \quad X \in \mathcal{X}, \quad C \in \mathbb{R}, \quad i \in I .
$$

In the case $\mathcal{X}=\mathcal{L}^{p}(\Omega), \mathbb{P}[e=1]=1,(10)$ coincides with cash invariance property (U1) of monetary utility functions. The interpretation is similar: $U_{i}(X)=\sup \{C \mid X-C \cdot e \in \mathcal{A}\}$, where $\mathcal{A}$ is an acceptance set, i.e. $U_{i}(X)$ represents the amount of "money" in terms of numeraire $e$ that one can consume and still stay in the acceptance set. Monetary utility functions, mean-deviation functionals (6), chance-constraint utilities (7), and mean-drawdown utilities (9) are all special cases of (10).

The next two propositions are central to the cash-invariant case.

Proposition 2 Given (10), an $\mathcal{F}$-feasible allocation $\mathbf{Y}=\left(Y_{1}, \ldots, Y_{m}\right)$ is $\mathcal{F}$-Pareto optimal if and only if

(i) $X^{*}=\sum_{i=1}^{m} Y_{i}$ is a maximizer to the investment problem

$$
\sup _{X \in \mathcal{F}} U^{*}(X)
$$

and

(ii) $U^{*}\left(X^{*}\right)=\sum_{i=1}^{m} U_{i}\left(Y_{i}\right)$,

where

$$
U^{*}(X) \equiv \sup _{\mathbf{Z} \in \mathcal{A}(X)} \sum_{i=1}^{m} U_{i}\left(Z_{i}\right) \quad \text { with } \quad \mathcal{A}(X)=\left\{\mathbf{Z}: \sum_{i=1}^{m} Z_{i}=X\right\} .
$$

Proof Let (i) and (ii) hold. Then $\sum_{i=1}^{m} U_{i}\left(Y_{i}\right)=U^{*}\left(X^{*}\right) \geqslant U^{*}\left(Z^{*}\right) \geqslant \sum_{i=1}^{m} U_{i}\left(Z_{i}\right)$ for any $\mathcal{F}$-feasible $\mathbf{Z}=\left(Z_{1}, \ldots, Z_{m}\right)$ with $Z^{*}=\sum_{i=1}^{m} Z_{i}$, so that $\mathbf{Y}$ is $\mathcal{F}$-Pareto optimal. Conversely, if either (i) or (ii) does not hold, there is an $\mathcal{F}$-feasible $\mathbf{Z}=\left(Z_{1}, \ldots, Z_{m}\right)$ with $\sum_{i=1}^{m} U_{i}\left(Z_{i}\right)-\sum_{i=1}^{m} U_{i}\left(Y_{i}\right)=\epsilon>0$. Then allocation $\mathbf{Z}^{\prime}=\left(Z_{1}^{\prime}, \ldots, Z_{m}^{\prime}\right)$ with $Z_{i}^{\prime}=Z_{i}+\left(U_{i}\left(Y_{i}\right)-U_{i}\left(Z_{i}\right)+\epsilon / m\right) e, i \in I$, is $\mathcal{F}$-feasible, and $U_{i}\left(Z_{i}^{\prime}\right)>U_{i}\left(Y_{i}\right)$, $i \in I$.

Proposition 3 Given (10), let $\mathbf{Y}=\left(Y_{1}, \ldots, Y_{m}\right)$ be any $\mathcal{F}$-Pareto optimal allocation. Then

(i) For any constant vector $\mathbf{C}=\left(C_{1}, \ldots C_{m}\right)$ with $\sum_{i=1}^{m} C_{i}=0$, the allocation

$$
\left(Y_{1}+C_{1} \cdot e, \ldots, Y_{m}+C_{m} \cdot e\right)
$$

is Pareto optimal; and 
(ii) All $\mathcal{F}$-Pareto optimal allocations (up to equivalence) can be represented in the form (13).

Proof Statement (i) follows from Proposition 2 and the relations $\sum_{i=1}^{m}\left(Y_{i}+C_{i} \cdot e\right)=\sum_{i=1}^{m} Y_{i}$ and $\sum_{i=1}^{m} U_{i}\left(Y_{i}+\right.$ $\left.C_{i} \cdot e\right)=\sum_{i=1}^{m} U_{i}\left(Y_{i}\right)$. Let us prove (ii). $\sum_{i=1}^{m} U_{i}\left(Z_{i}\right)=U^{*}\left(Z^{*}\right)=U^{*}\left(Y^{*}\right)=\sum_{i=1}^{m} U_{i}\left(Y_{i}\right)$ for any other $\mathcal{F}$ Pareto optimal allocation $\mathbf{Z}=\left(Z_{1}, \ldots, Z_{m}\right)$ with $Z^{*}=\sum_{i=1}^{m} Z_{i}$, where the first and second equalities follow from statements (ii) and (i) of Proposition 2. Consequently, $\mathbf{Z}$ is equivalent to (13) with $C_{i}=U_{i}\left(Z_{i}\right)-U_{i}\left(Y_{i}\right)$, $i \in I$.

Propositions 2 and 3 imply that the cooperative investment problem (1) with cash-invariant utilities (10) can be solved in three independent steps.

\section{Algorithm 1 (Cooperative investment with cash-invariant utilities)}

(S1) Finding an optimal portfolio. Find solution $X^{*}$ to problem (11). If $X^{*}$ is not unique, choose any.

(S2) Optimal allocation. Divide $X^{*}$ among the agents to maximize the sum of agents' utilities. If resulting allocation $\mathbf{Y}=\left(Y_{1}, \ldots, Y_{m}\right)$ is not unique, choose any. Regardless of the choices made at steps $S 1$ and $S 2$, the set of all $\mathcal{F}$-Pareto optimal allocations (up to equivalence) is given by (13).

(S3) Fair capital reallocation. Choose C in (13) such that the resulting $\mathcal{F}$-Pareto optimal allocation is "fair."

The notion of "fairness" in step S3 is not universal. For example, in a mutual fund, the management, being a participant of the fund, attracts customers by promising them a modest improvement in their utilities, so that it collects the rest (major part) of the fund's profit. What is important in Algorithm 1 is that steps S1 and S2 are not affected by how "fairness" is defined. In other words, regardless of how the optimal investment is divided, it should always solve problem (12), and the sum of agent's utilities should be maximized, otherwise the resulting allocation will not be $\mathcal{F}$-Pareto optimal. Sections 3.1, 3.2 and 3.3 discuss steps S1, S2 and S3, respectively.

\subsection{Finding an Optimal Portfolio}

Step S1 in Algorithm 1 solves problem (11) with the cooperative utility function $U^{*}$, which under (10) is cashinvariant. If all $U_{i}$ are concave, then $U^{*}$ is also concave. If, in addition, $\mathcal{X}=\mathcal{L}^{p}(\Omega)$ and at least one of $U_{i}$ is monotone, then $U^{*}$ is monotone (see [18, Proposition 10]), and consequently, $U^{*}$ is a monetary utility function. For optimality conditions for (11) with monetary utility functions and for corresponding optimization methods, see e.g. [29].

Grechuk et al. [17] showed that if $\mathcal{X}=\mathcal{L}^{2}(\Omega)$ and $U_{i}$ are mean-deviation functionals (6) for some deviation measures $\mathcal{D}_{i}$ and constants $d_{i}>0, i \in I$, then $U^{*}$ can also be represented by (6) with the deviation measure

$$
\mathcal{D}^{*}(X)=\inf _{Y \in \mathcal{A}(X)} \max \left\{\mathcal{D}_{1}\left(Y_{1}\right) / d_{1}, \ldots, \mathcal{D}_{m}\left(Y_{m}\right) / d_{m}\right\},
$$

where $\mathcal{A}(X)$ is defined as in (12). In this case, (11) reduces to mean-deviation optimization

$$
\inf _{X \in \mathcal{F}, E[X] \geqslant \pi} \mathcal{D}^{*}(X) \Longleftrightarrow \sup _{X \in \mathcal{F}, \mathcal{D}^{*}(X) \leqslant d_{0}} E[X] .
$$

Rockafellar et al. [34, 35] derived optimality conditions for (15) in a single-period setting, whereas $\mathrm{Li} \& \mathrm{Ng}$ [26] and Li \& Zhou [27] solved (15) with $\mathcal{D}^{*}=\sigma$ in multiperiod and continuous-time settings, respectively.

Propositions 2 and 3 hold for non-concave utility functions, e.g. for VaR.

Example 9 Let $\Omega$ be atomless and let $\mathcal{X}=\mathcal{L}^{p}(\Omega), I=\{1,2\}, \alpha_{i} \in(0,1), i \in I$, and $\alpha_{1}+\alpha_{2}<1$. Then

(i) If $U_{i}(X)=-\operatorname{VaR}_{\alpha_{i}}(X), i \in I$, then $U^{*}(X)=-\operatorname{VaR}_{\alpha_{1}+\alpha_{2}}(X)$;

(ii) If $U_{i}$ are given by (7) with $\alpha_{i}$ and $\Delta_{i}, i \in I$, then $U^{*}$ is also given by (7) with $\alpha=\alpha_{1}+\alpha_{2}, \Delta=\Delta_{1}+\Delta_{2}$. 
Detail. (i) Let $X=Y_{1}+Y_{2}$. Then $P\left[X \leqslant y_{1}+y_{2}\right] \leqslant P\left[Y_{1} \leqslant y_{1}\right]+P\left[Y_{2} \leqslant y_{2}\right]$ for all $y_{1}, y_{2} \in \mathbb{R}$, and by definition, $q_{\alpha_{1}+\alpha_{2}}(X) \geqslant q_{\alpha_{1}}\left(Y_{1}\right)+q_{\alpha_{2}}\left(Y_{2}\right)$, which implies $U^{*}(X) \leqslant-\operatorname{VaR}_{\alpha_{1}+\alpha_{2}}(X)$. Let us prove the reverse inequality. Since $\Omega$ is atomless, there exist disjoint sets $A, B$, and $C$ such that $A \cup B \cup C=\Omega$, $\mathbb{P}[A]=\alpha_{1}, \mathbb{P}[B]=\alpha_{2}$, and $X(\omega) \leqslant q_{\alpha_{1}}(X)$ for all $\omega \in A, q_{\alpha_{1}}(X) \leqslant X(\omega) \leqslant q_{\alpha_{1}+\alpha_{2}}(X)$ for all $\omega \in B$, and $q_{\alpha_{1}+\alpha_{2}}(X) \leqslant X(\omega)$ for all $\omega \in C$. Let $Y_{1}(\omega)$ be defined by $X-\left(q_{\alpha_{1}+\alpha_{2}}(X)-q_{\alpha_{1}}(X)\right)$ for $\omega \in A$ and by $q_{\alpha_{1}}(X)$ for $\omega \notin A$, and let $Y_{2}=X-Y_{1}$. Then $\mathbb{P}\left[Y_{1} \geqslant q_{\alpha_{1}}(X)\right] \geqslant 1-\mathbb{P}[A]=1-\alpha_{1}$, so that $q_{\alpha_{1}}\left(Y_{1}\right) \geqslant q_{\alpha_{1}}(X)$. Also, $\mathbb{P}\left[Y_{2} \geqslant q_{\alpha_{1}+\alpha_{2}}(X)-q_{\alpha_{1}}(X)\right] \geqslant \mathbb{P}[A]+\mathbb{P}[C]=1-\alpha_{2}$, and consequently, $q_{\alpha_{2}}\left(Y_{2}\right) \geqslant q_{\alpha_{1}+\alpha_{2}}(X)-q_{\alpha_{1}}(X)$, which yields $q_{\alpha_{1}}\left(Y_{1}\right)+q_{\alpha_{2}}\left(Y_{2}\right) \geqslant q_{\alpha_{1}+\alpha_{2}}(X)$, and thus, (i) follows.

In (ii), $U^{*}(X)=E[X]$ if and only if there exist $Y_{1}, Y_{2} \in \mathcal{L}^{p}(\Omega)$ such that $X=Y_{1}+Y_{2}$ and $\operatorname{VaR}_{\alpha_{i}}\left(Y_{i}-\right.$ $\left.E\left[Y_{i}\right]\right) \leqslant \Delta_{i}, i \in I$. By virtue of (i), the last condition is equivalent to $\operatorname{VaR}_{\alpha_{1}+\alpha_{2}}(X-E[X]) \leqslant \Delta_{1}+\Delta_{2}$.

Example 10 Let $\mathcal{X}=\mathcal{L}^{p}(\Omega), I=\{1,2\}$, and let $U_{i}$ be given by (7) with $\alpha_{i} \in(0,1)$ and $\Delta_{i}>0, i \in I$, such that $\alpha_{1}+\alpha_{2}<1 / 2$ and that $\mathcal{F}=\{x \cdot r \mid x \in \mathbb{R}\}$, where $r$ is an r.v. uniformly distributed on $[a, b]$. Then,

(i) For agent $i$, a solution to (3) is $X_{i}^{*}=x_{i}^{*} \cdot r$ with $x_{i}^{*}=\frac{\Delta_{i}}{(b-a)\left(\frac{1}{2}-\alpha_{i}\right)}$ and $U_{i}\left(X_{i}^{*}\right)=E\left[X_{i}^{*}\right]=\frac{\Delta_{i}}{1-2 \alpha_{i}}$.

(ii) A solution to (11) is $X^{*}=x^{*} \cdot r$ with $x^{*}=\frac{\Delta_{1}+\Delta_{2}}{(b-a)\left(\frac{1}{2}-\left(\alpha_{1}+\alpha_{2}\right)\right)}$ and $U^{*}\left(X^{*}\right)=\frac{\Delta_{1}+\Delta_{2}}{1-2\left(\alpha_{1}+\alpha_{2}\right)}>U_{1}\left(X_{1}^{*}\right)+$ $U_{2}\left(X_{2}^{*}\right)$.

(iii) If $\Delta_{1}=\Delta_{2}=\Delta$ and $\alpha_{1}=\alpha_{2}=1 / 5$, then $U_{1}\left(X_{1}^{*}\right)=U_{2}\left(X_{2}^{*}\right)=5 \Delta / 3$ and $U^{*}\left(X^{*}\right)=10 \Delta=$ $3\left(U_{1}\left(X_{1}^{*}\right)+U_{2}\left(X_{2}^{*}\right)\right)$.

Detail. For agent $i, U_{i}(x \cdot r)<\infty$ if $\operatorname{VaR}_{\alpha_{i}}(x \cdot r-x \cdot E r) \leqslant \Delta_{i}$, or, equivalently, if $-\left(a+(b-a) \alpha_{i}\right) x+$ $\frac{a+b}{2} x \leqslant \Delta_{i}$, which implies $x \leqslant \frac{\Delta_{i}}{(b-a)\left(1 / 2-\alpha_{i}\right)}$, and (i) follows. By a similar argument, Example 9 implies (ii).

If (11) has no solution, there are no $\mathcal{F}$-Pareto optimal allocations. For $\epsilon>0$, we say that $\mathbf{Z} \epsilon$-dominates $\mathbf{Y}$ if $U_{i}\left(Z_{i}\right) \geqslant U_{i}\left(Y_{i}\right), i \in I$, with $U_{i}\left(Z_{i}\right) \geqslant U_{i}\left(Y_{i}\right)+\epsilon$ for some $i \in I$. An $\mathcal{F}$-feasible allocation $\mathbf{Y}$ is $(\mathcal{F}, \epsilon)$ Pareto optimal, if no $\mathcal{F}$-feasible allocation $\epsilon$-dominates $Y$. A slight modification of the proofs of Propositions 2 and 3 imply that if $U^{*}\left(X^{*}\right)>U^{*}(X)-\epsilon / 2$ for all $X \in \mathcal{F}$ and $\sum_{i=1}^{m} U_{i}\left(Y_{i}\right)>U^{*}\left(X^{*}\right)-\epsilon / 2$ for some $\mathbf{Y} \in \mathcal{A}\left(X^{*}\right)$, then $\mathbf{Y}$ is $(\mathcal{F}, \epsilon)$-Pareto optimal, and so are all allocations given by (13). In particular, this implies that $(\mathcal{F}, \epsilon)$-Pareto optimal allocations exist for all $\epsilon>0$ if and only if

$$
\sup _{X \in \mathcal{F}} U^{*}(X)<+\infty
$$

If (16) does not hold, there are $\mathcal{F}$-feasible allocations $Y$ with $U_{i}\left(Y_{i}\right)>C, i \in I$, for every $C \in \mathbb{R}$.

\subsection{Optimal Allocation}

Now we assume that (16) holds and that problem (11) does have a solution $X^{*}$. Step S2 in Algorithm 1 is to find an allocation $\mathbf{Y} \in \mathcal{A}\left(X^{*}\right)$ such that $\sum_{i=1}^{m} U_{i}\left(Y_{i}\right)=U^{*}\left(X^{*}\right)$.

A typical allocation (risk sharing) problem is formulated as follows. Agent $i \in I$ has a utility function $U_{i}: \mathcal{X} \rightarrow[-\infty, \infty)$ and an initial endowment $Q_{i} \in \mathcal{X}$ such that $U_{i}\left(Q_{i}\right)>-\infty$. The problem is completely determined by the triplet $\mathcal{P}_{C}=(\mathcal{X}, \mathbf{Q}, \mathbf{U})$, where $\mathbf{Q}=\left(Q_{1}, \ldots, Q_{m}\right)$ and $\mathbf{U}=\left(U_{1}, \ldots, U_{m}\right)$. An allocation $\mathbf{Y}=\left(Y_{1}, \ldots, Y_{m}\right)$ is $\mathbf{Q}$-feasible if $\sum_{i=1}^{m} Y_{i}=\sum_{i=1}^{m} Q_{i}$. A $\mathbf{Q}$-feasible allocation $\mathbf{Y}$ is $\mathbf{Q}$-Pareto optimal, if no $\mathbf{Q}$-feasible allocation dominates $\mathbf{Y}$. Proposition 2 can be reformulated as follows.

Proposition 4 Given (10), an $\mathcal{F}$-feasible allocation $\mathbf{Y}=\left(Y_{1}, \ldots, Y_{m}\right)$ is $\mathcal{F}$-Pareto optimal if and only if

(i) $X^{*}=\sum_{i=1}^{m} Y_{i}$ is a maximizer in (11), and 
(ii) $\mathbf{Y}$ is $\mathbf{Q}$-Pareto optimal for some (and hence for every) $\mathbf{Q}$ such that $\sum_{i=1}^{m} Q_{i}=X^{*}$.

Thus, Step 2 reduces to finding a Q-Pareto optimal allocation, which is well studied for several frequently used families of utility functions on $\mathcal{L}^{p}(\Omega)$, including those satisfying (10) (see, e.g. [23] for monetary utility functions, and [21] for mean-deviation functionals (6)). In particular, Jouini et.al. [23] proved the existence of Q-Pareto optimal allocation for monetary utility functions if $p=\infty$ and $\Omega$ is atomless. Here, we prove a more general existence result.

Proposition 5 Let $\mathcal{U}_{i}: \mathcal{L}^{p}(\Omega) \rightarrow[-\infty, \infty), i=1, \ldots, m$ be monetary utility functions consistent with SSD, let $X \in \mathcal{L}^{p}(\Omega)$, and let (16) hold. Then

(i) the supremum in (12) is attained;

(ii) for every $\mathbf{Q}$ such that $\sum_{i=1}^{m} Q_{i}=X$, there exists a $\mathbf{Q}$-Pareto optimal allocation $\mathbf{Y}$, such that all $Y_{i}$ are pairwise comonotone.

Proof It follows from Proposition 1 in [28] that (12) can be rewritten as

$$
U^{*}(X)=\sup _{\mathbf{Y} \in \mathcal{C}(X)} \sum_{i=1}^{m} U_{i}\left(Y_{i}\right)
$$

where $\mathcal{C}(X)$ is the set of all allocations $\mathbf{Y}$ such that $\sum_{i=1}^{m} Y_{i}=X$ and that all $Y_{i}$ are pairwise comonotone. Let us prove that the supremum in (17) is attained, and both (i) and (ii) follow. This statement is trivial if $U^{*}(X)=-\infty$, so let $\mathcal{U}^{*}(X) \in \mathbb{R}$. With (U1), equation (17) can be rewritten as

$$
U^{*}(X)=E[X]+\sup _{\mathbf{Z} \in \mathcal{C}^{\prime}(\bar{X})} \sum_{i=1}^{m} U_{i}\left(Z_{i}\right),
$$

where $\bar{X}=X-E[X]$ and $\mathcal{C}^{\prime}(\bar{X})$ is the set of all allocations $\mathbf{Z}$ such that $Z_{i}$ are pairwise comonotone, $\sum_{i=1}^{m} Z_{i}=$ $\bar{X}$ and $E\left[Z_{i}\right]=0, i=1, \ldots, m$.

For r.v.'s $T$ and $S$, we write $T \succeq_{c v} S$ if $E[T]=E[S]$ and $T \succeq_{2} S$, or, equivalently, if

$$
\int_{0}^{\alpha} q_{T}(t) d t \geqslant \int_{0}^{\alpha} q_{S}(t) d t, \quad \alpha \in[0,1]
$$

with equality for $\alpha=1$, see [14, Theorem 2.57]. First, we show that $Z_{i} \succeq_{c v} \bar{X}$ for every $\mathbf{Z} \in \mathcal{C}^{\prime}(\bar{X})$ and every $i=1, \ldots, m$. By contradiction, let $\int_{0}^{\alpha} q_{Z_{i}}(t) d t<\int_{0}^{\alpha} q_{\bar{X}}(t) d t$ for some $i$ and some $\alpha \in(0,1)$. Then $\int_{0}^{\alpha} q_{Z}(t) d t=\int_{0}^{\alpha} q_{\bar{X}}(t) d t-\int_{0}^{\alpha} q_{Z_{i}}(t) d t>0$, where $Z=\bar{X}-Z_{i}$, and the equality follows from comonotonicity of $Z_{i}$ and $Z$. Since $q_{Z}(t)$ is non-decreasing, this implies that $q_{Z}(\alpha)>0$ and $\int_{\alpha}^{1} q_{Z}(t) d t \geqslant(1-\alpha) q_{Z}(\alpha) \geqslant$ 0 , so that $E[Z]=\int_{0}^{1} q_{Z}(t) d t>0$. However, $E[Z]=E\left[\bar{X}-Z_{i}\right]=0$, which is a contradiction.

Because $Z \succeq_{c v} \bar{X}$ implies that $\|Z\|_{p} \leqslant\|\bar{X}\|_{p}$, the set $\mathcal{Z}=\left\{Z \in \mathcal{L}^{p}(\Omega) \mid Z \succeq_{c v} \bar{X}\right\}$ is a bounded closed convex subset of $\mathcal{L}^{p}(\Omega)$. If $1<p<\infty, \mathcal{L}^{p}(\Omega)$ is a reflexive Banach space, and, consequently, $\mathcal{Z}$ is a compact set in a weak topology, i.e., every sequence in $\mathcal{Z}$ has a convergent subsequence. Let $\left\{\mathbf{Z}^{n}\right\}$ be a maximizing sequence in (18). By passing to subsequence, if necessary, we may assume that each component sequence $\left\{Z_{i}^{n}\right\}, i=1, \ldots, m$ converges weakly to some r.v. $Z_{i}^{*} \in \mathcal{Z}$. Because every monetary utility function $\mathcal{U}_{i}$, $i=1, \ldots, m$, is concave and upper-semicontinuous, it is also upper-semicontinuous in the weak topology, i.e. $U_{i}\left(Z_{i}^{*}\right) \geqslant \limsup _{n \rightarrow \infty} U_{i}\left(Z_{i}^{n}\right)$. Thus, $\sum_{i=1}^{m} U_{i}\left(Z_{i}^{*}\right) \geqslant \sum_{i=1}^{m} \limsup _{n \rightarrow \infty} U_{i}\left(Z_{i}^{n}\right) \geqslant \liminf _{n \rightarrow \infty} \sum_{i=1}^{m} U_{i}\left(Z_{i}^{n}\right)=U^{*}(\bar{X})$, where the last inequality holds since $\left\{\mathbf{Z}^{n}\right\}$ is a maximizing sequence in (18). Because $Z^{*}=\left(Z_{1}^{*}, \ldots, Z_{n}^{*}\right) \in$ $\mathcal{C}^{\prime}(\bar{X})$, this implies that the infimum in (18) is attained, and the statement holds for $1<p<\infty$. 
If $p=1$, then $\mathcal{L}^{1}(\Omega)$ is not reflexive and a separate proof of weak compactness of $\mathcal{Z}$ is required. By Dunford-Pettis theorem [7, Chapter II, Theorem T25], a weakly closed subset $\mathcal{Z}$ of $\mathcal{L}^{1}(\Omega)$ is weakly compact if it is uniformly integrable, i.e.

$$
\lim _{K \rightarrow \infty} \sup _{Z \in \mathcal{Z}} E\left[|Z| I_{\{|Z| \geqslant K\}}\right]=0,
$$

where $I$ is the indicator function equal to 1 if the condition in curly brackets holds and equal to zero otherwise. Let us prove (19). We have $E\left[|Z| I_{\{|Z| \geqslant K\}}\right]=E\left[-Z I_{\{Z \leqslant-K\}}\right]+E\left[Z I_{\{Z \geqslant K\}}\right]$, and

$$
K \alpha_{Z}(K) \leqslant E\left[-Z I_{\{Z \leqslant-K\}}\right]=-\int_{0}^{\alpha_{Z}(K)} q_{Z}(t) d t \leqslant-\int_{0}^{\alpha_{Z}(K)} q_{\bar{X}}(t) d t
$$

where $\alpha_{Z}(K)=\mathbb{P}[Z \leqslant-K]$, and the last inequality follows from $Z \succeq_{c v} \bar{X}$. Thus, for any $\epsilon>0$, one may guarantee $\alpha_{Z}(K)<\epsilon$ for sufficiently large $K$, and consequently, $E\left[-Z I_{\{Z \leqslant-K\}}\right] \leqslant-\int_{0}^{\epsilon} q_{\bar{X}}(t) d t$. Since the last expression is independent of $Z$ and vanishes as $\epsilon \rightarrow 0$, and $E\left[Z I_{\{Z \geqslant K\}}\right]$ can be bounded similarly, (19) follows. Thus, $\mathcal{Z}$ is weakly compact, and the rest of the argument is the same as for the case of $1<p<\infty$.

Finally, let $p=\infty$, and let $\left\{\mathbf{Z}^{n}\right\}$ be a maximizing sequence in (18). Since $Z_{i}^{n}$ and $\bar{X}$ are comonotone, we may assume that $Z_{i}^{n}=f_{i}^{n}(\bar{X}), n \in \mathbb{N}, i=1, \ldots, m$, for some non-decreasing functions $f_{i}^{n}$ defined on $[\inf \bar{X}, \sup \bar{X}]$. Fix $i \in\{1, \ldots, m\}$. In view of $Z_{i}^{n} \succeq_{c v} \bar{X}, \inf \bar{X} \leqslant Z_{i}^{n}(\omega) \leqslant \sup \bar{X}$ holds with probability 1 , and, consequently, the functions $f_{i}^{n}, n \in \mathbb{N}$, are uniformly bounded. Since $\sum_{i=1}^{m} f_{i}^{n}(x)=x$ for all $x, f_{i}^{n}(x)-$ $f_{i}^{n}(y)|\leqslant| x-y \mid$ for all $x$ and $y$, so the functions $f_{i}^{n}, n \in \mathbb{N}$, form a bounded uniformly equicontinuous family. Thus, by the Arzela-Ascoli theorem [25], the sequence $f_{i}^{n}, n \in \mathbb{N}$, has a subsequence that uniformly converges to some $f_{i}^{*}$. By passing to subsequence, if necessary, we may assume that $\lim _{n \rightarrow \infty} Z_{i}^{n}=Z_{i}^{*}:=f_{i}^{*}(\bar{X}) \in \mathcal{L}^{\infty}(\Omega)$. Then $U_{i}\left(Z_{i}^{*}\right) \geqslant \limsup _{n \rightarrow \infty} U_{i}\left(Z_{i}^{n}\right)$ by upper-semicontinuity of $U_{i}$, and the rest of the proof is the same as in the case of $1<p<\infty$.

\subsection{Fair Capital Reallocation}

Now suppose that step S2 in Algorithm 1 yields an allocation $\mathbf{Y}$ such that $\sum_{i=1}^{m} U_{i}\left(Y_{i}\right)=U^{*}\left(X^{*}\right)$. Then all $\mathcal{F}$-Pareto optimal allocations (up to equivalence) are given by (13). This section addresses step S3 in Algorithm 1: choosing $\mathbf{C}$ in (13) such that the resulting allocation is "fair."

Let $\mathbf{Q}$ be any set of initial endowments such that $\sum_{i=1}^{m} Q_{i}=X^{*}$. Then the set of $\mathcal{F}$-Pareto optimal allocations is exactly the set of $\mathbf{Q}$-Pareto optimal allocations in a typical allocation (risk sharing) problem $\mathcal{P}_{C}=(\mathcal{X}, \mathbf{Q}, \mathbf{U})$. A "fair" element in Pareto optimal set for $\mathcal{P}_{C}$ can be identified based on the notion of equilibrium. A price $P$ is any non-zero linear continuous functional on $\mathcal{X}$. A pair $(\mathbf{Z}, P)$ is $\mathbf{Q}$-equilibrium, if two conditions hold: (i) $\mathbf{Z}$ is $\mathbf{Q}$-feasible and (ii) for every $i \in I, Z_{i}$ solves the problem

$$
\sup _{X \in \mathcal{X}} U_{i}(X) \quad \text { subject to } P(X)=P\left(Q_{i}\right) .
$$

If $P(e)=0$ for $e$ defined in (10), problem (21) has no solution, which is a contradiction. Thus, without loss of generality, we assume $P(e) \neq 0$ and rescale $P$ such that $P(e)=1$. The constraint $P(X)=P\left(Q_{i}\right)$ in (21) is then equivalent to $P(X) \leqslant P\left(Q_{i}\right)$. If $(\mathbf{Z}, P)$ is $\mathbf{Q}$-equilibrium, $P$ and $\mathbf{Z}$ will be called $\mathbf{Q}$-equilibrium price and Q-equilibrium allocation, respectively. The first welfare theorem [3] states that under mild conditions on $U_{i}$, e.g. (10) suffices, every $\mathbf{Q}$-equilibrium allocation is $\mathbf{Q}$-Pareto optimal. Usually, $\mathbf{Q}$-equilibrium allocations are considered as "fair" elements of Q-Pareto optimal set.

Proposition 6 Given (10), let $\mathbf{Y}$ be $\mathbf{Q}$-Pareto optimal allocation, and let $X^{*}=\sum_{i=1}^{m} Y_{i}$. Then

(i) $P$ is a Q-equilibrium price if and only if $P \in \partial U^{*}\left(X^{*}\right)$, where $\partial U^{*}\left(X^{*}\right) \equiv\left\{P \mid U^{*}(X)-U^{*}\left(X^{*}\right) \leqslant\right.$ $P\left(X-X^{*}\right)$ for all $\left.X \in \mathcal{X}\right\}$ is the super-differential of $U^{*}$ at $X^{*}$; 
(ii) for every $P \in \partial U^{*}\left(X^{*}\right)$, (13) is a $\mathbf{Q}$-equilibrium allocation if and only if

$$
C_{i}=P\left(Q_{i}-Y_{i}\right), \quad i \in I .
$$

Proof (i) Let $(\mathbf{Z}, P)$ be a $\mathbf{Q}$-equilibrium, $X \in \mathcal{X}$, and $\mathbf{X}=\left(X_{1}, \ldots, X_{m}\right) \in \mathcal{A}(X)$. Then $P\left(X_{i}^{\prime}\right)=P\left(Z_{i}\right)$ for $X_{i}^{\prime}=X_{i}+\left(P\left(Z_{i}\right)-P\left(X_{i}\right)\right) e, i \in I$, which implies $U_{i}\left(X_{i}^{\prime}\right) \leqslant U_{i}\left(Z_{i}\right), i \in I$, or $\sum_{i=1}^{m} U_{i}\left(X_{i}\right)-P(X)+$ $P\left(X^{*}\right) \leqslant \sum_{i=1}^{m} U_{i}\left(Z_{i}\right) \leqslant U^{*}\left(X^{*}\right)$. The supremum over $\mathbf{X} \in \mathcal{A}(X)$ yields $U^{*}(X)-P(X)+P\left(X^{*}\right) \leqslant$ $U^{*}\left(X^{*}\right)$, or, equivalently, $P \in \partial U^{*}\left(X^{*}\right)$, and the "only if" part follows. Conversely, if $P \in \partial U^{*}\left(X^{*}\right)$ then $P(C \cdot e) \geqslant U^{*}\left(X^{*}+C \cdot e\right)-U^{*}\left(X^{*}\right)=C$ for all $C \in \mathbb{R}$, which implies $P(e)=1$, and the "if" part follows from (ii).

(ii) Necessity of (22) is obvious, and sufficiency is proved as follows. Let $U_{1}\left(X_{1}^{*}\right)>U_{1}\left(Y_{1}+P\left(Q_{1}-\right.\right.$ $\left.Y_{1}\right)$ e) for some $X_{1}^{*} \in \mathcal{X}$ such that $P\left(X_{1}^{*}\right)=P\left(Q_{1}\right)$. Then

$$
U^{*}(X) \geqslant U_{1}\left(X_{1}^{*}\right)+\sum_{i=2}^{m} U_{i}\left(Y_{i}+P\left(Q_{i}-Y_{i}\right) e\right)>\sum_{i=1}^{m} U_{i}\left(Y_{i}+P\left(Q_{i}-Y_{i}\right) e\right)=U^{*}\left(X^{*}\right),
$$

and $P(X)=P\left(X_{1}^{*}\right)+\sum_{i=2}^{m} P\left(Q_{i}\right)=P\left(X^{*}\right)$ for $X=X_{1}^{*}+\sum_{i=2}^{m}\left(Y_{i}+P\left(Q_{i}-Y_{i}\right) e\right)=U\left(X^{*}\right)$, which contradicts with the assumption that $P \in \partial U^{*}\left(X^{*}\right)$.

Proposition 6 suggests a "fair" allocation from the $\mathbf{Q}$-Pareto optimal set in the problem $\mathcal{P}_{C}=(\mathcal{X}, \mathbf{Q}, \mathbf{U})$. In particular, if the set $\partial U^{*}\left(X^{*}\right)$ is a singleton, (22) determines the unique allocation (up to equivalence). For monetary utility functions on $\mathcal{X}=\mathcal{L}^{\infty}(\Omega)$, this result is due to Filipovic and Kupper [13, Theorem 3.13], and for mean-deviation functionals (6), it is proved in [21]. However, monetary utility functions and mean-deviation functionals are concave, whereas Proposition 6 requires utility functions to be only cash-invarinat.

Observe that the capital reallocation (22) depends on the choice of initial endowments $\mathbf{Q}$. In fact, any $\mathcal{F}$-Pareto optimal allocation $\mathbf{Y}$ can be made $\mathbf{Q}$-equilibrium by taking, say, $\mathbf{Q}=\mathbf{Y}$. However, if $U^{*}$ is concave, the following result holds.

Proposition 7 Given (10), suppose the following conditions hold: $\mathcal{F}$ is a convex set; $U^{*}$ is concave on $\mathcal{X}$ and is finite and continuous at some point $X_{0} \in \mathcal{F} ; Y$ is an $\mathcal{F}$-Pareto optimal allocation; and $X^{*}=\sum_{i=1}^{m} Y_{i}$. Then

(i) there exists a price $P^{*} \in \partial U^{*}\left(X^{*}\right)$ such that $P^{*}(X) \leqslant P^{*}\left(X^{*}\right)$ for all $X \in \mathcal{F}$;

(ii) in particular, if $\mathcal{F}$ is a subspace of $\mathcal{X}, P^{*}(X)=0$ for all $X \in \mathcal{F}$.

Proof Because $\mathbf{Y}$ is $\mathcal{F}$-Pareto optimal, $U^{*}\left(X^{*}\right)<\infty$, and the part (i) follows from [38, Theorem 2.9.1]. If $\mathcal{F}$ is a subspace of $\mathcal{X}$, then $P^{*}\left(X^{*}\right) \geqslant P^{*}(X)=2 P^{*}\left(X^{*}\right)-P^{*}\left(2 X^{*}-X\right) \geqslant 2 P^{*}\left(X^{*}\right)-P^{*}\left(X^{*}\right)=P^{*}\left(X^{*}\right)$, or, equivalently, $P^{*}(X)=P^{*}\left(X^{*}\right)=P^{*}(0)=0$ for all $X \in \mathcal{F}$.

Part (ii) in Proposition 7 implies that (22) with $P=P^{*}$ determines the same capital reallocation $C_{i}=$ $P^{*}\left(-Y_{i}\right), i \in I$, for any choice of initial endowments $\mathbf{Q}$ such that $Q_{i} \in \mathcal{F}, i \in I$. As in Proposition 6, if the set $\partial U^{*}\left(X^{*}\right)$ is a singleton, (22) provides the unique element (up to equivalence) from the $\mathcal{F}$-Pareto optimal set (13).

The cooperative investment problem (1) with cash-invariant $\mathbf{U}$ (satisfying (10)) can be viewed as a cooperative game with transferable payoffs with the set of players $I$ and with the value $v(S)$ of every coalition $S \subset I$ assigned by

$$
v(S)=\sup _{X \in \mathcal{F}} U_{S}(X), \quad \text { where } \quad U_{S}(X) \equiv \sup _{\mathcal{A}_{S}(X)} \sum_{i \in S} U_{i}\left(Z_{i}\right) \quad \text { and } \quad \mathcal{A}_{S}(X) \equiv\left\{\mathbf{Z}: \sum_{i \in S} Z_{i}=X\right\} .
$$

In particular, for $S=\{i\}, v(S)$ is the utility $U_{i}$ of the optimal individual investment (3).

Vector $y=\left(y_{1}, \ldots, y_{m}\right) \in \mathbb{R}^{m}$ belongs to the core of the game (23) if and only if $\sum_{i=1}^{m} y_{i}=v(I)$ and $\sum_{i \in S} y_{i} \geqslant v(S)$ for every $S \subset I$, see [32]. 
Proposition 8 Given (10), suppose the following conditions hold: $\mathrm{Y}$ is an $\mathcal{F}$-Pareto optimal allocation; $X^{*}=$ $\sum_{i=1}^{m} Y_{i}$; and $P^{*} \in \partial U^{*}\left(X^{*}\right)$ is a price such that $P^{*}(X)=0$ for all $X \in \mathcal{F}$. Then $y=\left(y_{1}, \ldots, y_{m}\right)$ with $y_{i}=U_{i}\left(Y_{i}-P^{*}\left(Y_{i}\right) e\right), i \in I$, belongs to the core of the game (23).

Proof The relation $\sum_{i=1}^{m} y_{i}=v(I)$ follows from Proposition 2 and $\mathcal{F}$-Pareto optimality of $\mathbf{Y}$. It is left to show $\sum_{i \in S} y_{i} \geqslant v(S)$ for all $S \subset I$. Let $\mathbf{Q} \in \mathcal{A}\left(X^{*}\right)$ be such that $P^{*}\left(Q_{i}\right)=0, i \in I$, e.g. $Q_{i}=X^{*} / m$, $i \in I$. Then $\mathbf{Y}$ is $\mathbf{Q}$-Pareto optimal by Proposition 4, and consequently, $\mathbf{Y}^{\prime}$ with $Y_{i}^{\prime}=Y_{i}-P^{*}\left(Y_{i}\right) e$ is a $\mathbf{Q}$ equilibrium allocation by Proposition 6. Since $P^{*}\left(Z-P^{*}(Z) e\right)=P^{*}\left(Y_{i}-P^{*}\left(Y_{i}\right) e\right)=P^{*}\left(Q_{i}\right)=0$, this implies $U_{i}\left(Y_{i}-P^{*}\left(Y_{i}\right) e\right) \geqslant U_{i}\left(Z-P^{*}(Z) e\right)$ for all $i \in I$ and $Z \in \mathcal{X}$. Thus,

$$
\sum_{i \in S} y_{i}=\sum_{i \in S} U_{i}\left(Y_{i}-P^{*}\left(Y_{i}\right) e\right) \geqslant \sum_{i \in S} U_{i}\left(Z_{i}-P^{*}\left(Z_{i}\right) e\right)=\sum_{i \in S} U_{i}\left(Z_{i}\right)-P^{*}\left(\sum_{i \in S} Z_{i}\right)=\sum_{i \in S} U_{i}\left(Z_{i}\right),
$$

for any $\mathbf{Z} \in \mathcal{A}_{S}(X)$ and any $X \in \mathcal{F}$. The supremum of this inequality over all $\mathbf{Z} \in \mathcal{A}_{S}(X)$ and all $X \in \mathcal{F}$ yields $\sum_{i \in S} y_{i} \geqslant v(S)$ for all $S \subset I$.

For monetary utility functions and mean-deviation functionals (6) (particular cases of cash-invariant utilities), Proposition 8 was proved in [13, Corollary 3.14] ${ }^{10}$ and [17], respectively.

\subsection{Summary of Cash-Invariant Case}

Propositions 2 and 3 show that with the cash-invariance assumption (10), the cooperative investment problem (1) can be solved in three independent steps formalized in Algorithm 1. Steps S1 and S2 are reduced to well-studied problems. Step S3 introduces a linear continuous pricing functional $P^{*}: \mathcal{X} \rightarrow \mathbb{R}$ such that (i) $P^{*}(e)=1$, (ii) $P^{*} \in \partial U^{*}\left(X^{*}\right)$, where $X^{*}=\sum_{i=1}^{m} Y_{i}$, and (iii) $P^{*}(X)=0$ for all $X \in \mathcal{F}$, and then determines capital reallocation as $C_{i}=P^{*}\left(-Y_{i}\right), i \in I$. Here, property (i) is normalization and property (ii) ensures that $P^{*}$ is an equilibrium price in any allocation problem $\mathcal{P}_{C}=(\mathcal{X}, \mathbf{Q}, \mathbf{U})$ such that the initial endowments $Q_{i}$ sum up to $X^{*}$. Finally, property (iii) ensures that the suggested capital reallocation is independent of the choice of $\mathbf{Q}$, at least if $Q_{i} \in \mathcal{F}, i \in I$. Another interpretation of property (iii) is that the "internal" price $P^{*}$ should be consistent with the "real" market price. Part (ii) in Proposition 7 provides the sufficient conditions for the existence of $P^{*}$, whereas Proposition 8 justifies the suggested capital reallocation from the game-theoretic perspective. If equilibrium pricing functional $P^{*}$ is not unique, but can be chosen arbitrary from a convex set, one may argue that "fair" capital reallocation should be done on the base of $P^{*}$ which is the "center" of this set, see [16].

\section{Arbitrary Utility Functions}

Here, we address the cooperative investment problem (1) with arbitrary utility functions. The main difference from the cash-invariant case (10) is that, in general, there is no "optimal cooperative investment" $X^{*}$ for which all $\mathcal{F}$-Pareto optimal allocations $\mathbf{Y}$ (up to equivalence) satisfy $\sum_{i=1}^{m} Y_{i}=X^{*}$. Thus, the agents cannot decide on their investment strategy before they agree on the method for choosing a "fair" allocation from $\mathcal{F}$-Pareto optimal set, i.e. the problem of optimal investment and allocation become inseparable.

The results in Section 3.3 suggest the following notion of "fairness." A pair $(\mathbf{Z}, P)$, where $\mathbf{Z}$ is an allocation and $P$ is a price functional, will be called $\mathcal{F}$-equilibrium, if (i) $\mathbf{Z}$ is $\mathcal{F}$-feasible and (ii) for every $i \in I, Z_{i}$ solves the problem

$$
\sup _{X \in \mathcal{X}} U_{i}(X) \quad \text { subject to } \quad P(X) \leqslant 0 .
$$

\footnotetext{
${ }^{10}$ The work [13] considers no optimal cooperative investment.
} 
In this case, $P$ and $Z$ will be called $\mathcal{F}$-equilibrium price and $\mathcal{F}$-equilibrium allocation, respectively. If $U_{i}, i \in I$, have no local maxima, ${ }^{11}$ then $P\left(Z_{i}\right)=0, i \in I$, for any $\mathcal{F}$-equilibrium allocation $\mathbf{Z}$. A pricing functional $P$ will be called consistent if $P(X)=0$ for all $X \in \mathcal{F}$. By fair allocation for problem (1) will be meant an allocation $\mathbf{Z}$ such that $(\mathbf{Z}, P)$ is an $\mathcal{F}$-equilibrium for some consistent pricing functional $P$.

The following proposition is an analogue of the first welfare theorem [3] for optimal investment.

Proposition 9 Suppose $U_{i}, i \in I$, have no local maxima. Then every fair allocation $\mathbf{Z}$ for problem (1) is $\mathcal{F}$-Pareto optimal.

Proof Let $P$ be a consistent pricing functional such that $(\mathbf{Z}, P)$ is an $\mathcal{F}$-equilibrium, and let $\mathbf{Y}$ be an $\mathcal{F}$-feasible allocation that dominates $\mathbf{Z}$. Then $U_{i}\left(Y_{i}\right)>U_{i}\left(Z_{i}\right)$, so that $P\left(Y_{i}\right)>0$ for some $i \in I$, and consequently, $P\left(Y_{j}\right)<0$ for some $j \in I$. Then since $P$ is continuous, there exists an open set $A$ such that $Y_{j} \in A$ and $P(X)<0$ for all $X \in A$. But the no-local-maxima assumption implies that $U_{j}(X)>U_{j}\left(Y_{j}\right)$ for some $X \in A$, so that $U_{j}\left(Z_{j}\right)>U_{j}\left(Y_{j}\right)$, which is a contradiction.

If (10) holds, then consistent $P$ is an $\mathcal{F}$-equilibrium pricing functional if and only if $P \in \partial U^{*}\left(X^{*}\right)$, where $U^{*}$ is given by (12) and $X^{*}$ solves (11). Sufficient conditions for the existence of such $P$ are given in part (ii) of Proposition 7. For arbitrary utilities, the existence of a consistent $\mathcal{F}$-equilibrium pricing functional can be studied using the connection between the investment problem (1), with $\mathcal{F}$ being a closed subspace of (locally convex topological vector space) $\mathcal{X}$, and the allocation problem from Section 3.2.

Elements of $\mathcal{F}$ correspond to zero-price instruments (portfolios), and consequently, an investor with endowment $Y \in \mathcal{X}$ can construct a portfolio $X \in \mathcal{X}$, whenever $X-Y \in \mathcal{F}$. This implies an equivalence relation $X \sim Y \leftrightarrow X-Y \in \mathcal{F}$ on $\mathcal{X}$. Since $\sim$ is reflexive, symmetric and transitive, it induces equivalence classes $[X]=\{Y \in \mathcal{X} \mid Y \sim X\}$, which form the quotient space $\mathcal{X} / \mathcal{F}$ : a vector space with operations $[X]+[Y]=[X+Y]$ and $\alpha[X]=[\alpha X]$ for $X, Y \in \mathcal{X}$ and $\alpha \in \mathbb{R}$. The quotient of a locally convex space by a closed subspace is locally convex as well. Also, if $\mathcal{X}$ is a Banach space with norm $\|\cdot\|$, then so is $\mathcal{X} / \mathcal{F}$ with the norm $\|[X]\|^{*} \equiv \inf _{Y \in \mathcal{F}}\|X-Y\|$.

The investment problem $\mathcal{P}=(\mathcal{X}, \mathcal{F}, \mathbf{U})$ can be put in correspondence to the allocation problem $\mathcal{P}^{*}=$ $\left(\mathcal{X} / \mathcal{F}, \mathbf{0}, \mathbf{U}^{*}\right)$, where $\mathbf{0}=([0], \ldots,[0])$ and $\mathbf{U}^{*}=\left(U_{1}^{*}, \ldots, U_{m}^{*}\right)$ with $U_{i}^{*}([X])=\sup _{Y \in \mathcal{F}} U_{i}(X+Y), i \in I$. An allocation $\mathbf{Y}$ is $\mathcal{F}$-feasible if and only if the corresponding allocation $[\mathbf{Y}]=\left(\left[Y_{1}\right], \ldots,\left[Y_{m}\right]\right)$ is $\mathbf{0}$-feasible in $\mathcal{P}^{*}$. Also, $\mathbf{Y}$ is $\mathcal{F}$-Pareto optimal if and only if $[\mathbf{Y}]$ is $\mathbf{0}$-Pareto-optimal in $\mathcal{P}^{*}$. Any consistent pricing functional $P: \mathcal{X} \rightarrow \mathbb{R}$ satisfies $P(X)=P(Y)$ whenever $X \sim Y$ and, thus, defines a pricing functional $P^{*}$ on $\mathcal{X} / \mathcal{F}$ by $P^{*}([X])=P(X) . P$ is a consistent $\mathcal{F}$-equilibrium pricing functional if and only if $P^{*}$ is a $\mathbf{0}$-equilibrium one.

The correspondence between the problems $\mathcal{P}$ and $\mathcal{P}^{*}$ allows results available for $\mathcal{P}^{*}$ to be applied to $\mathcal{P}$. Next proposition is a result of this correspondence.

Proposition 10 There exists a fair allocation in $\mathcal{P}$ if and only if there exists a $\mathbf{0}$-equilibrium in $\mathcal{P}^{*}$.

Proposition 10 reduces the problem of fair allocation to the well-studied problem on the existence of $\mathbf{Q}$ equilibrium in $\mathcal{P}^{*}$ with different utility functions. In particular, Theorem $1 \mathrm{in}$ [12] proves that a $\mathbf{Q}$-equilibrium ${ }^{12}$ exists if the following assumptions hold

(A1) $\mathcal{X}_{i} \equiv\left\{X \mid U_{i}^{*}(X)>-\infty\right\}, i \in I$, are convex, closed, and nonempty.

(A2) If $\mathbf{Y}$ is $\mathbf{Q}$-feasible and $P_{i}(X) \equiv\left\{X^{\prime} \in \mathcal{X} / \mathcal{F} \mid U_{i}^{*}\left(X^{\prime}\right)>U_{i}^{*}(X)\right\}$ is the preferred set of agent $i \in I$ at $X$, then $P_{i}\left(Y_{i}\right) \neq \varnothing, i \in I$, and int $P_{1}\left(Y_{1}\right) \neq \varnothing$.

\footnotetext{
${ }^{11} U_{i}$ has no local maxima if for all $X \in \mathcal{X}$ and $A \subset \mathcal{X}$ such that $A$ is open and $X \in A$, there exists $X^{\prime} \in A$ such that $U_{i}\left(X^{\prime}\right)>U_{i}(X)$.

${ }^{12}$ In fact, Theorem 1 in [12] proves the existence of quasiequilibrium, i.e. a pair $\left(\mathbf{Z}, P^{*}\right)$ such that (i) $\mathbf{Z}$ is $\mathbf{Q}$-feasible, (ii) $P^{*}\left(Z_{i}\right)=$ $P^{*}\left(Q_{i}\right), i \in I$, and (iii) $P^{*}(X) \geqslant P^{*}\left(Q_{i}\right)$, whenever $U_{i}^{*}(X)>U_{i}^{*}\left(Z_{i}\right), i \in I$. A quasiequilibrium is a $Q$-equilibrium if $P^{*}\left(Z_{i}\right)>$ $\inf _{X \in \mathcal{X}_{i}} P^{*}(X), i \in I$.
} 
(A3) $U_{i}^{*}, i \in I$, are strictly quasi-concave.

(A4) For a $\mathbf{Q}$-feasible allocation $\mathbf{Y}$, the set $\mathcal{U}=\left\{\left(z_{1}, \ldots, z_{m}\right) \in \mathbb{R}^{m} \mid U_{i}^{*}\left(Q_{i}\right) \leqslant z_{i} \leqslant U_{i}^{*}\left(Y_{i}\right), i \in I\right\}$ is compact.

If A1-A3 hold, Theorem 1 in [12] can be loosely stated as "A Q-equilibrium exists if $\mathcal{U}$ is compact." The set $\mathcal{U}$ is known to be compact for expected utilities [6] and for law-invariant monetary utility functions [11, Proposition 4].

\section{Investment with Drawdown Risk Measure}

This section finds an optimal investment strategy subject to a constraint on investment drawdown (8). Let $\mathcal{X}$ be a space of stochastic processes $X=X_{t}, t \in \mathcal{T}$, with either $\mathcal{T}=\left\{t_{1}, \ldots, t_{k}=T\right\}$ or $\mathcal{T}=[0, T]$. Elements $X \in \mathcal{X}$ are cumulative portfolio gains, and $\mathcal{F} \subset \mathcal{X}$ corresponds to feasible portfolios. Let $I=\{1,2\}$, and let utility functions $U_{i}$ be given by (9) with $\alpha_{i} \in[0,1]$ and $C_{i}>0$ for $i \in I$, where $\alpha_{1}>\alpha_{2}$. Because $U_{i}$ are translation invariant, Proposition 2 holds, and the goal is to characterize $U^{*}$ in (11). In this case, the following statement holds.

Proposition 11 Let $X \in \mathcal{X}$, and let $d(X)$ be drawdown of $X$. Also, let $d_{1}$ and $d_{2}$ be nonnegative r.v's on $\Omega \times \mathcal{T}$ such that $d(X)=d_{1}+d_{2}$. Then there exist $Y_{1}, Y_{2} \in \mathcal{X}$ such that $X=Y_{1}+Y_{2}$ and $d_{i}=d\left(Y_{i}\right), i \in I$. In particular, for any constant $C \in \mathbb{R}$, one can take $Y_{1}=C-d_{1}, Y_{2}=X+d_{1}-C$.

Proof For any $\omega \in \Omega$,

$$
d_{\omega, t_{\min }}(X)=\sup _{\tau \leqslant t_{\min }} X_{\tau}(\omega)-X_{t_{\min }}(\omega)=X_{t_{\min }}(\omega)-X_{t_{\min }}(\omega)=0,
$$

where $t_{\min }=t_{1}$ if $\mathcal{T}=\left\{t_{1}, \ldots, t_{k}=T\right\}$, and $t_{\min }=0$ if $\mathcal{T}=[0, T]$. Hence, $d_{1}\left(\omega, t_{\min }\right)=d_{2}\left(\omega, t_{\min }\right)=0$, and

$$
\left.d_{\omega, t}\left(Y_{1}\right)=d_{\omega, t}\left(-d_{1}\right)=\sup _{\tau \leqslant t}\left(-d_{1}(\omega, \tau)\right)+d_{1}(\omega, t)=-d_{1}\left(\omega, t_{\min }\right)\right)+d_{1}(\omega, t)=d_{1}(\omega, t) \quad \forall \omega, t .
$$

Also,

$$
\begin{aligned}
d\left(Y_{2}\right) & =\sup _{\tau \leqslant t}\left[X_{\tau}(\omega)+d_{1}(\tau, \omega)\right]-\left[X_{t}(\omega)+d_{1}(t, \omega)\right]=\sup _{\tau \leqslant t}\left[X_{\tau}(\omega)\right]-X_{t}(\omega)-d_{1}(t, \omega) \\
& =d(X)-d_{1}=d_{2},
\end{aligned}
$$

holds for all $t \in \mathcal{T}$ and $\omega \in \Omega$, where the second equality is equivalent to simultaneously holding of " $\geqslant$ " and " $\leqslant$, with " $\geqslant$ " coming from nonnegativity of $d_{1}$ and with " $\leqslant$ " coming from $X_{\tau}(\omega)+d_{1}(\tau, \omega) \leqslant X_{\tau}(\omega)+$ $d_{\tau, \omega}(X)=\sup _{\tau^{\prime} \leqslant \tau}\left[X_{\tau^{\prime}}(\omega)\right] \leqslant \sup _{\tau \leqslant t}\left[X_{\tau}(\omega)\right]$ for $\tau \leqslant t$.

Example 11 (cooperative investment with drawdown constraints) The individual investment problems are to maximize $U_{i}$ given by (9) with $\alpha_{1}>\alpha_{2}, \alpha_{i} \in[0,1]$, and $C_{i}>0$ for $i=1,2$. The cooperative investment problem (11) takes the form

$$
\begin{aligned}
& \sup _{X \in \mathcal{F}, a \geqslant 0} E[X] \\
& \text { subject to } \frac{1}{1-\alpha_{1}} \int_{\alpha_{1}}^{1} \min \left\{q_{s}(d(X)), a\right\} d s \leqslant C_{1} \text { and } \frac{1}{1-\alpha_{2}} \int_{\alpha_{2}}^{1}\left[q_{s}(d(X))-a\right]_{+} d s \leqslant C_{2},
\end{aligned}
$$

and Pareto optimal allocations are given by $Y_{1}=C-\min \left\{d\left(X^{*}\right), a^{*}\right\}, Y_{2}=X^{*}-Y_{1}$, where $C \in \mathbb{R}$ is arbitrary, and $X^{*} \in \mathcal{F}$ and $a^{*} \geqslant 0$ are optimal solutions in (26). 
Detail. Proposition 11 with $d_{1}=\min \left\{d\left(X^{*}\right), a^{*}\right\}$ and $d_{2}=\left[d\left(X^{*}\right)-a^{*}\right]_{+}$shows that $d\left(Y_{i}\right)=d_{i}, i \in I$, and the constraints in (26) imply that

$$
\mathrm{CDaR}_{\alpha_{i}}\left(Y_{i}\right)=\frac{1}{1-\alpha_{i}} \int_{\alpha_{i}}^{1} q_{s}\left(d\left(Y_{i}\right)\right) d s=\frac{1}{1-\alpha_{i}} \int_{\alpha_{i}}^{1} q_{s}\left(d_{i}\right) d s \leqslant C_{i}, \quad i \in I .
$$

Thus, $Y_{1}$ and $Y_{2}$ form a feasible division. Let us prove the converse: if $\mathrm{CDaR}_{\alpha_{i}}\left(Z_{i}\right) \leqslant C_{i}, i \in I$, for some $Z_{1}, Z_{2} \in \mathcal{X}$, then the constraints in (26) are satisfied for $X=Z_{1}+Z_{2}$ and some $a \geqslant 0$. This is obvious if $\mathrm{CDaR}_{\alpha_{1}}(X)<C_{1}$ (in this case, the first constraint holds for all $a$, and the second one is satisfied for sufficiently large $a$ ), otherwise we can choose $a$ such that the first constraint holds as equality. Let $\alpha^{\prime}$ be such that $q_{s}(d(X)) \leqslant a$ for $\alpha<\alpha^{\prime}$ and $q_{s}(d(X)) \geqslant a$ for $\alpha>\alpha^{\prime}$. Also, let $\alpha^{*}$ be defined as $\alpha^{*}=\alpha_{2}$ if $\alpha^{\prime}<\alpha_{2}$, $\alpha^{*}=\alpha_{1}$ if $\alpha^{\prime}>\alpha_{1}$, and $\alpha^{*}=\alpha^{\prime}$ otherwise. Then

$$
\int_{\alpha^{*}}^{1} \min \left\{q_{s}(d(X)), a\right\} d s \geqslant \int_{\alpha^{*}}^{1} q_{s}\left(d\left(Z_{1}\right)\right) d s .
$$

Indeed, if $\alpha^{*}=\alpha_{1}$, then (27) reduces to $\mathrm{CDaR}_{\alpha_{1}}\left(Z_{1}\right) \leqslant C_{1}$. Otherwise $\alpha_{1} \geqslant \alpha^{*} \geqslant \alpha^{\prime}$, and

$$
\int_{\alpha^{*}}^{1} \min \left\{q_{s}(d(X)), a\right\} d s=\int_{\alpha^{*}}^{1} a d s=\left(1-\alpha^{*}\right) C_{1} \geqslant \frac{1-\alpha^{*}}{1-\alpha_{1}} \int_{\alpha_{1}}^{1} q_{s}\left(d\left(Z_{1}\right)\right) d s \geqslant \int_{\alpha^{*}}^{1} q_{s}\left(d\left(Z_{1}\right)\right) d s,
$$

where the second equality and the last inequality follow from $C_{1}=\frac{1}{1-\alpha_{1}} \int_{\alpha_{1}}^{1} a d s=a$ and monotonicity of the quantile function, respectively. Finally,

$$
\begin{aligned}
\int_{\alpha_{2}}^{1}\left[q_{s}(d(X))-a\right]_{+} d s & =\int_{\alpha^{*}}^{1}\left[q_{s}(d(X))-a\right]_{+} d s=\int_{\alpha^{*}}^{1} q_{s}(d(X)) d s-\int_{\alpha^{*}}^{1} \min \left\{q_{s}(d(X)), a\right\} d s \\
& \leqslant \int_{\alpha^{*}}^{1} q_{s}\left(d\left(Z_{2}\right)\right) d s \leqslant \int_{\alpha_{2}}^{1} q_{s}\left(d\left(Z_{2}\right)\right) d s \leqslant\left(1-\alpha_{2}\right) C_{2},
\end{aligned}
$$

where the first inequality follows from (27) and subadditivity of $\mathrm{CDaR}_{\alpha^{*}}$, see [8, 9, 39].

If the probability space is discrete and $\mathcal{F}$ is determined by a set of linear constraints, then as in $[8,9,39]$, problem (26) can be reduced to linear programming.

As an illustration, Example 11 is specialized for the case of a single risky instrument with a two-period binomial tree model. At each period, the return of the risky instrument assumes values $a$ and $-b$ with probabilities $p$ and $q=1-p$, respectively, where $a>b>0$ and $p a-q b>0$. Also, an agent can decide not to invest (zero return). In this case, $\mathcal{X}$ is a space of stochastic processes $X=X_{t}, t \in \mathcal{T}$, where $\mathcal{T}=\left\{t_{1}, t_{2}\right\}$, and $\mathcal{F}$ is a finite subset of $\mathcal{X}$ corresponding to all possible "trading strategies" (there are 6 of them ${ }^{13}$ ). Agent 1 maximizes $E\left[X_{2}\right]$ subject to $\operatorname{MaxDD}(X) \leqslant b\left(\alpha \rightarrow 1\right.$ and $C=b$ in (9)), whereas agent 2 maximizes $E\left[X_{2}\right]$ subject to $\operatorname{AvgDD}(X) \leqslant b q^{2} / 2\left(\alpha=0\right.$ and $C=b q^{2} / 2$ in (9)). For agent 1 , the optimal strategy is to invest at the first period and in case of "win" invest again, whereas in the case of "loss" not to invest. The expected return of agent 1 is then $(p a-q b)+p(p a-q b)$. For agent 2, the only feasible strategy is not to invest at all, and thus, to have zero return. However, if the agents cooperate, they can invest at both periods, so that agent 2 gains 0 after the first period and then gains $a, 0$, and $-b$ in the cases of win-win, win-loss (or loss-win), and loss-loss, respectively. Then the average drawdown for agent 2 is 0 except for the case of loss-loss, so that $\operatorname{AvgDD}\left(Y_{1}\right)=b q^{2} / 2$, whereas the constraint $\operatorname{MaxDD}\left(Y_{2}\right) \leqslant b$ for agent 1 is also satisfied. The cooperative investment expected return is $2(p a-q b)>(p a-q b)+p(p a-q b)$.

\footnotetext{
13 (1) "invest," “invest”; (2) "invest," "not invest"; (3) "not invest," “invest"; (4) "not invest," "not invest"; (5) "invest," and then if "win," "invest" (if "lose," "not invest"); and (6) "invest," and then if "lose," "invest" (if "win," "not invest").
} 


\section{Conclusions}

Cooperative investment aims to form an optimal cooperative portfolio and to share the portfolio profit/loss among participating agents fairly. Agents' utility functions and a feasible investment set are assumed to be arbitrary, whereas investment strategies can be single-period and multiperiod. The cooperative portfolio problems have first been solved for the case of cash-invariant utilities: (i) the set of all $\mathcal{F}$-Pareto optimal solutions has been characterized, (ii) sufficient conditions for the existence of a "fair" element from $\mathcal{F}$-Pareto-optimal set have been established, and (iii) if exists, the "fair" element has been proved to be in the core of the corresponding cooperative game. Similar results were previously obtained only in some special cases: in the context of expected utility theory [37], for mean-deviation functionals [17], and for singleton $\mathcal{F}$ [13]. Then the method of choosing a "fair" $\mathcal{F}$-Pareto-optimal allocation has been extended to the case of arbitrary utility functions: an allocation $\mathbf{Z}$ such that $(\mathbf{Z}, P)$ is an $\mathcal{F}$-equilibrium for some consistent pricing functional $P$ has been suggested, and existence of such $\mathbf{Z}$ has been discussed. The corresponding pricing functional $P$ can also be used for pricing of contingent claims in incomplete markets. The obtained results have been demonstrated for cooperative investment with agents using drawdown constraints in multiperiod investment strategies.

\section{Acknowledgment}

The authors are grateful to the referees for their comments and suggestions, which helped to improve the quality of the paper. The first author also thanks the University of Leicester for granting him the academic study leave to conduct this research.

\section{References}

[1] Aase, K. (2002): Perspectives of risk sharing. Scandinavian Actuarial Journal 2, 73-128.

[2] Acciaio, B. (2007): Optimal risk sharing with non-monotone monetary functionals. Finance and Stochastics 11(2), 267-289

[3] Becker, R. (1991): Fundamental theorems of welfare economics in infinite dimensional commodity spaces. In: Equilibrium Theory in Infinite Dimensional Spaces, Ali Khan, M. and Yannelis N., ed. Studies in Economic Theory, 1, Springer, 124-175.

[4] Burgert, C., Ruschendorf, L. (2008): Allocation of risks and equilibrium in markets with finitely many traders. Insurance: Mathematics and Economics 42(1), 177-188.

[5] Cerreia-Vioglio, S., Maccheroni, F., Marinacci, M., Montrucchio, L. (2011): Risk measures: rationality and diversification, Mathematical Finance 21, 743-774.

[6] Cheng, H. (1991): Asset market equilibrium in infinite dimensional complete markets. Journal of Mathematical Economic 20, 137-152.

[7] Dellacherie, C. and Meyer, P.A. (1978): Probabilities and Potential, North-Holland Pub. Co, N. Y.

[8] Chekhlov, A., Uryasev, S., Zabarankin, M. (2003): Portfolio optimization with drawdown constraints, in Asset and Liability Management Tools, ed. B. Scherer (Risk Books, London), 263-278.

[9] Chekhlov, A., Uryasev, S., Zabarankin, M. (2005): Drawdown measure in portfolio optimization, International Journal of Theoretical and Applied Finance 8(1), 13-58.

[10] Cvitanic, J., Karatzas, I. (1995): On portfolio optimization under "drawdown" constraints, IMA Lecture Notes in Mathematics \& Applications 65, 77-88. 
[11] Dana, R.-A. (2011): Comonotonicity, efficient risk-sharing and equilibria in markets with short-selling for concave law-invariant utilities. Journal of Mathematical Economics 47(3), 328-335.

[12] Dana, R.-A., Le Van C., Magnien F. (1997): General equilibrium in asset markets with or without shortselling. Journal of Mathematical Analysis and Applications 206(2), 567-588.

[13] Filipovic D., Kupper M. (2008): Equilibrium prices for monetary utility functions, International Journal of Theoretical and Applied Finance 11, 325-343.

[14] Follmer, H., Schied, A. (2011): Stochastic finance, (3nd ed.). Berlin New York: de Gruyter.

[15] Gilboa, I., Samuelson, L. and Schmeidler, D. (2014): No-Betting-Pareto Dominance, Econometrica, 82, $1405-1442$.

[16] Grechuk, B. (2015): The center of a convex set and capital allocation, European Journal of Operational Research 243(2), 628-636.

[17] Grechuk, B., Molyboha, A., Zabarankin, M. (2013): Cooperative games with general deviation measures, Mathematical Finance 23(2), 339-365.

[18] Grechuk, B., Molyboha, A., Zabarankin, M. (2012): Mean-deviation analysis in the theory of choice, Risk Analysis 32(8), 1277-1292.

[19] Grechuk B., Zabarankin, M. (2015): Inverse portfolio problem with coherent risk measures. European Journal of Operational Research, to appear, DOI:10.1016/j.ejor.2015.09.050

[20] Grechuk B., Zabarankin, M. (2014): Inverse portfolio problem with mean-deviation model. European Journal of Operational Research 234(2), 481-490.

[21] Grechuk, B., Zabarankin, M. (2012): Optimal risk sharing with general deviation measures, Annals of Operations Research 200(1), 9-21.

[22] Grossman, S. J., Zhou, Z. (1993): Optimal investment strategies for controlling drawdowns, Mathematical Finance 3(3), 241-276.

[23] Jouini, E., Schachermayer, W., Touzi, N. (2008): Optimal risk sharing for law invariant monetary utility functions, Mathematical Finance 18(2), 269-292.

[24] Kiesel, S. and Ruschendorf, L. (2014): Optimal risk allocation for convex risk functionals in general risk domains, Statistics \& Risk Modeling 31(3-4), 335-365.

[25] Kurdila, A. and Zabarankin, M. (2005) Convex Functional Analysis, Birkhäuser.

[26] Li, D., Ng, W.-L. (2000): Optimal dynamic portfolio selection: multiperiod mean-variance formulation, Mathematical Finance 10, 387-406.

[27] Li, D., Zhou, X.Y. (2000): Continuous-time mean-variance portfolio selection: A stochastic LQ framework, Applied Mathematics and Optimization 42, 19-33.

[28] Ludkovski, M., Ruschendorf, L. (2008): On comonotonicity of Pareto optimal risk sharing. Statistics and Probability Letters 78(10), 1181-1188.

[29] Luthi, H., Doege, J. (2005): Convex risk measures for portfolio optimization and concepts of flexibility. Mathematical Programing, Ser. B 104(2-3), 541-559. 
[30] Markowitz, H.M. (1952): Portfolio selection. Journal of Finance 7(1), 77-91.

[31] Muthuraman, K. and Kumar, S. (2006): Multidimensional portfolio optimization with proportional transaction costs. Mathematical Finance 16(2), 301-335.

[32] Osborne, J., Rubinstein, A. (1994): A course in game theory. Cambridge, MA: MIT Press.

[33] Rockafellar, R.T., Uryasev, S., Zabarankin, M. (2006): Generalized deviations in risk analysis. Finance \& Stochastics 10(1), 51-74.

[34] Rockafellar, R.T., Uryasev, S., Zabarankin, M. (2006): Optimality conditions in portfolio analysis with general deviation measures. Mathematical Programming 108(2-3), 515-540.

[35] Rockafellar, R.T., Uryasev, S., Zabarankin, M. (2006): Master funds in portfolio analysis with general deviation measures. The Journal of Banking and Finance 30(2), 743-77.

[36] Wilson, R. (1968): The theory of syndicates. Econometrica 36(1), 119-132.

[37] Xia, J. (2004): Multi-agent investment in incomplete markets. Finance and Stochastics 8(2), 241-259.

[38] Zalinescu, C. (2002): Convex analysis in general vector spaces, World Scientific, Singapore.

[39] Zabarankin, M., Pavlikov, K., Uryasev, S. (2014): Capital asset pricing model (CAPM) with drawdown measure, European Journal of Operational Research 234(2), 508-517. 